\title{
Multi-object spectroscopy of low-redshift EIS clusters
}

\section{Reliability of matched-filter results at $z \sim 0.3-0.4^{\star}, \star \star$}

\author{
L. F. Grove ${ }^{1, \star \star \star}$, L. da Costa ${ }^{2}$, and C. Benoist ${ }^{3}$ \\ 1 Dark Cosmology Centre, Niels Bohr Institute, University of Copenhagen, Juliane Maries Vej 30, 2100 Copenhagen, Denmark \\ e-mail: lisbeth@dark-cosmology.dk \\ 2 Observatório Nacional, Rua Gen. José Cristino 77, Rio de Janeiro, R.J., Brazil \\ 3 Laboratoire Cassiopée, CNRS, UMR 6202, Observatoire de la Côte d’Azur, BP 4229, 06304 Nice Cedex 4, France
}

Received 28 November 2006 / Accepted 16 September 2008

ABSTRACT

\begin{abstract}
This paper is the last in a series investigating low-redshift galaxy systems identified by the matched-filter technique in a moderately deep I-band survey. In this paper we present new redshifts for 747 galaxies in 23 ESO Imaging Survey (EIS) cluster fields. We use the "gap"-technique to search for significant overdensities in redshift space for identifying groups/clusters of galaxies corresponding to the original EIS matched-filter cluster candidates. In this way we spectroscopically confirm systems in 10 of the 23 cluster candidate fields with a matched-filter estimated redshift $z_{\mathrm{MF}}=0.3-0.4$ and with spectroscopic redshifts in the range from $z=0.158$ to $z=0.534$, with the observations favouring the confirmation of systems at the lower redshift end. After careful analysis of the redshift distribution, one system was split into two very close clumps in redshift space. We find that the systems identified in the present paper span a broad range of velocity dispersion and richness. The measured one-dimensional velocity dispersion range from $175 \mathrm{~km} \mathrm{~s}^{-1}$ to $497 \mathrm{~km} \mathrm{~s}^{-1}$, consistent with the values obtained in previous papers using much larger samples for systems over the same redshift range. Both undersampling and contamination by substructures contribute to the uncertainty of these measurements. The richness range corresponds to clusters with an estimated total luminosity in the range $12 L^{*}<L<65 L^{*}$, but these estimates are very uncertain as are their relation to the velocity dispersion (mass) of the systems. From the analysis of the colours of the galaxy populations we find that $\sim 60 \%$ of the spectroscopically confirmed systems have a "significant" red sequence. We find that the colour of the red sequence galaxies matches passive stellar evolution predictions. With this paper we complete our spectroscopic survey of the fields of 58 EIS cluster candidates with estimated redshifts $z_{\mathrm{MF}} \leq 0.4$. We have measured a total of 1954 galaxy redshifts in the range $z=0.0065$ to $z=0.6706$. Of the 58 systems we confirm $42(\sim 75 \%)$ with redshifts between $z=0.095$ and $z=0.534$.
\end{abstract}

Key words. galaxies: clusters: general - cosmology: observations - galaxies: distances and redshifts - galaxies: photometry

\section{Introduction}

Clusters of galaxies have long been recognised as important targets for cosmology and astrophysics, both to constrain cosmological parameters and for evolutionary studies of large-scale structure and of cluster members. However, to carry out these studies large samples of clusters of galaxies with well-defined selection criteria, representative of the entire population of clusters and spanning a wide redshift range are required.

Over the past decades a number of systematic efforts to assemble catalogues of galaxy clusters (e.g. Gunn et al. 1986; Postman et al. 1996; Scodeggio et al. 1999; Gladders \& Yee 2001; Gonzalez et al. 2001; Bahcall et al. 2003; Olsen et al. 2007; van Breukelen et al. 2006; Koester et al. 2007) have been undertaken based on large optical and infrared imaging surveys in one or more passbands, using different search techniques. This has greatly expanded the available samples both at low and high redshifts, as compared to those detected in X-rays (e.g. Rosati 1998; Böhringer et al. 2000). Unfortunately, systematic

^ Based on observations made with the Danish1.5-m telescope at ESO, La Silla, Chile.

$\star \star$ Full Table 3 is only available in electronic form at the CDS via anonymous ftp to cdsarc.u-strasbg.fr (130.79.128.5) or via http://cdsweb.u-strasbg.fr/cgi-bin/qcat?J/A+A/490/945

$\star \star \star$ L. F. Grove previously published under L. F. Olsen. studies determining the yield of real bound systems from these image based searches has not progressed as fast, mainly because spectroscopic data covering large areas of the sky are difficult to assemble especially at intermediate and high redshifts. Furthermore, each detection technique is based on some different characteristic of the cluster such as its luminosity function, galaxy population or gas content. Therefore, it is important to understand the impact that these underlying assumptions may have on the detection and on the estimates of redshift and richness. In order to be able to use cluster samples in statistical studies, especially to constrain cosmological parameters, it is important not only to detect the systems but also be able to establish how reliable estimates for the redshift and richness are, how well they relate to the underlying mass of the system and the selection function of the sample. To determine that requires both extensive spectroscopic follow-up and the use of realistic numerical simulations from which mock galaxy catalogues can be built. Here we continue the effort of analysing the ESO Imaging Survey (EIS) Cluster Candidate Catalogue constructed using a matched-filter algorithm (Olsen et al. 1999a,b; Scodeggio et al. 1999). A first estimate of the selection function for this catalogue based on simplistic simulations was given in Olsen (2000). In this paper we concentrate on the results of a comprehensive spectroscopic follow-up of these cluster candidates, covering a broad range of redshifts. While firm conclusions cannot be drawn due to the 
small size of the sample, the issues that may affect the use of galaxy clusters for statistical studies can be explored and will serve as a guide for further investigation based on on-going numerical simulations.

This paper is part of a series reporting the results obtained from a spectroscopic follow-up of selected cluster candidate fields drawn from the original EIS cluster candidate sample assembled by Olsen et al. (1999a,b) and Scodeggio et al. (1999). This sample was constructed from the EIS I-band survey over 17 square degrees of the sky using the matched-filter technique. Out of 302 identified cluster candidates we have so far studied more than 50 candidate cluster fields chosen to contain candidates at different estimated redshift bins. So far we have probed fields with candidates at low $\left(z_{\mathrm{MF}} \sim 0.2\right.$, Hansen et al. 2002; Olsen et al. 2003, 2005a, hereafter Papers I, II and III), intermediate $\left(0.5 \leq z_{\mathrm{MF}} \leq 0.7\right.$, Ramella et al. 2000) and high redshifts $\left(z_{\mathrm{MF}} \geq 0.6\right.$, Benoist et al. 2002; Olsen et al. 2005b) using different telescopes and spectrographs. In this paper we extend the previous work at the low-redshift end by carrying out spectroscopic observations of 23 fields around cluster candidates selected in the estimated redshift bin $0.3 \leq z_{\mathrm{MF}} \leq 0.4$.

The paper is structured as follows: Sect. 2 gives an overview of the observations and data reduction. Section 3 reviews the identification of systems in redshift space as well as the procedure adopted for associating the redshift groups to the EIS detections. Section 4 describes the photometric properties of the spectroscopically confirmed systems including an analysis of the colour properties of the galaxy populations. Finally, in Sect. 5 a brief discussion of the conclusions of the results obtained in the present paper is presented, followed by a summary of the results of the entire series in Sect. 6.

\section{Observations and data reduction}

In the present work, all cluster candidates with estimated redshifts in the range $0.3 \leq z_{\mathrm{MF}} \leq 0.4$ within EIS patches $\mathrm{A}$ and B covering an area of $\sim 3.5$ square degrees (Olsen et al. 1999a,b) were selected for investigation. A total of 23 fields were observed, one of which containing two cluster candidates. Unfortunately, one of them (EISJ2243-4008) was not properly sampled and is not included in the present analysis. We list the 23 targeted cluster fields in Table 1, giving in Col. 1 an identifier for the cluster field; in Col. 2 the name of the field referring to the notation adopted by Olsen et al. (1999a,b); in Cols. 3 and 4 the matched filter position in J2000; in Col. 5 the matched filter estimated redshift and in Col. 6 the $\Lambda_{\mathrm{cl} \text {,org }}$-richness, which is the original richness estimate of the matched filter detection. The candidates roughly cover a richness range corresponding to Abell richness classes $\leq 1$ (e.g. Postman et al. 1996).

The observations were carried out at the Danish $1.54 \mathrm{~m}$ telescope at La Silla, Chile. We used the Danish Faint Object Spectrograph and Camera (DFOSC) in the Multi-Object Spectroscopy (MOS)-mode. The field of view of DFOSC corresponds to $3.4 \mathrm{Mpc}$ and $4.1 \mathrm{Mpc}$ at $z=0.3$ and $z=0.4$ (assuming $H_{0}=75 \mathrm{~km} \mathrm{~s}^{-1} \mathrm{Mpc}^{-1}, \Omega_{\mathrm{m}}=0.3$ and $\Omega_{\Lambda}=0.7$ ). This is a good match to the typical size of a cluster. The effective field that could be covered with MOS slit masks was typically 12 '. $\times 6.5$, depending on the exact configuration of galaxy positions in each field. The slit width was set to $2^{\prime \prime}$, and the slit length varied according to the extent of each galaxy. We used grism \#4, giving a dispersion of $220 \AA / \mathrm{mm}$, and covering, on average, a wavelength range from 3800 to $7500 \AA$. The resolution, as determined from HeNe line spectra, was found to be $16.6 \AA$ FWHM.
Table 1. Cluster candidates selected for observations.

\begin{tabular}{rllllr}
\hline \hline ID & Field $^{a}$ & $\alpha_{J 2000}$ & $\delta_{J 2000}$ & $z_{\mathrm{MF}}$ & $\Lambda_{\mathrm{cl}, \mathrm{org}}$ \\
\hline 1 & EISJ0044-2950A & 004458.6 & -295049.5 & 0.3 & 23.1 \\
2 & EISJ0045-2944 & 004500.8 & -294457.7 & 0.4 & 35.5 \\
3 & EISJ0047-2942 & 004723.0 & -294259.0 & 0.4 & 30.4 \\
4 & EISJ0048-2928 & 004825.8 & -292850.1 & 0.4 & 36.6 \\
5 & EISJ0049-2920 & 004931.3 & -292034.1 & 0.3 & 35.7 \\
6 & EISJ2236-3935 & 223602.9 & -393533.7 & 0.3 & 44.5 \\
7 & EISJ2236-4026 & 223647.6 & -402617.4 & 0.4 & 44.0 \\
8 & EISJ2236-4014 & 223652.6 & -401453.0 & 0.4 & 37.4 \\
9 & EISJ2237-4000 & 223711.4 & -400016.1 & 0.3 & 31.3 \\
10 & EISJ2238-3934 & 223803.4 & -393450.4 & 0.3 & 41.8 \\
11 & EISJ2239-3954 & 223918.4 & -395434.9 & 0.3 & 62.5 \\
12 & EISJ2240-4021 & 224007.8 & -402108.0 & 0.3 & 41.2 \\
13 & EISJ2241-4006 & 224126.7 & -400624.7 & 0.3 & 32.6 \\
14 & EISJ2241-3932 & 224131.3 & -393210.4 & 0.4 & 44.5 \\
15 & EISJ2243-4010A & 224301.9 & -401024.8 & 0.3 & 39.1 \\
16 & EISJ2243-3952 & 224319.4 & -395241.2 & 0.3 & 50.9 \\
17 & EISJ2243-3959 & 224329.4 & -395933.5 & 0.3 & 45.0 \\
18 & EISJ2243-4010B & 224342.7 & -401030.4 & 0.3 & 32.4 \\
$-{ }^{b}$ & EISJ2243-4008 & 224347.4 & -400847.0 & 0.3 & 34.3 \\
19 & EISJ2243-3947 & 224356.1 & -394728.8 & 0.4 & 48.6 \\
20 & EISJ2244-4008 & 224421.8 & -400821.6 & 0.4 & 31.1 \\
21 & EISJ2244-4019 & 224428.4 & -401946.5 & 0.3 & 38.3 \\
22 & EISJ2246-4012B & 224648.5 & -401248.2 & 0.4 & 39.5 \\
23 & EISJ2248-4015 & 224854.8 & -401518.8 & 0.3 & 36.2 \\
\hline
\end{tabular}

${ }^{a}$ Here we have added a " $J$ " in the name to conform with international standards. The EIS identification is the same except for this " $J$ "; ${ }^{b}$ overlaps with EISJ2243-4014B and separate observations were not carried out.

For each field we created two slit masks for an exposure time of 1.5 h per mask. We estimate the $S / N$ of the spectra for which we could measure the redshift to be in the range 5 to 15 .

We preferentially targeted bright galaxies with $I$-magnitude $I \leq 20.0\left(\mathrm{Vega}^{1}\right)$. The Schechter magnitude is estimated to be $I^{*} \sim 18.5$ and 19.3 at $z=0.3$ and 0.4 , respectively, using an absolute Schechter magnitude in the Cousin filter of $M_{I}^{*}=-22.33$ as commonly adopted (e.g. Postman et al. 1996; Olsen et al. 1999a). The corresponding apparent magnitude was computed using the $K$-correction for an elliptical galaxy template spectrum from the Kinney library (Kinney et al. 1996). We thus estimate our survey to cover galaxies to between $\sim 1.5$ and $\sim 0.7$ mag fainter than the Schechter magnitude. The target galaxies were selected without applying any additional colour selection. This allows us to investigate whether systems without red sequences are present in our sample. The matched-filter sample does not have any built-in bias against such systems and therefore provides the necessary input for such an investigation.

The data reduction was performed using standard procedures available in $\operatorname{IRAF}^{2}$ packages as described in detail in previous papers of this series (in particular Papers I and III). The

\footnotetext{
1 All magnitudes are quoted in the EIS magnitude system as provided by the EIS team, see Nonino et al. (1999), Prandoni et al. (1999), Benoist et al. (1999).

2 IRAF is distributed by the National Optical Astronomy Observatories, which is operated by AURA Inc. under contract with NSF.
} 
Table 2. Summary of spectroscopic coverage for each target cluster.

\begin{tabular}{lccccc}
\hline \hline ID Field & $\begin{array}{c}\text { Covered } \\
\text { area }\end{array}$ & & & & \\
\hline 1 EISJ0044-2950A & 56.2 & 35 & 20 & 0.54 & 0.57 \\
2 EISJ0045-2944 & 60.4 & 40 & 32 & 0.67 & 0.80 \\
3 EISJ0047-2942 & 78.9 & 36 & 31 & 0.46 & 0.86 \\
4 EISJ0048-2928 & 75.8 & 38 & 26 & 0.50 & 0.68 \\
5 EISJ0049-2920 & 71.6 & 39 & 35 & 0.56 & 0.90 \\
6 EISJ2236-3935 & 123.4 & 52 & 42 & 0.39 & 0.81 \\
7 EISJ2236-4026 & 64.3 & 41 & 21 & 0.52 & 0.51 \\
8 EISJ2236-4014 & 76.4 & 42 & 28 & 0.52 & 0.68 \\
9 EISJ2237-4000 & 111.6 & 55 & 35 & 0.49 & 0.64 \\
10 EISJ2238-3934 & 118.0 & 55 & 45 & 0.38 & 0.82 \\
11 EISJ2239-3954 & 100.1 & 48 & 42 & 0.43 & 0.88 \\
12 EISJ2240-4021 & 102.0 & 48 & 36 & 0.48 & 0.75 \\
13 EISJ2241-4006 & 91.5 & 57 & 36 & 0.53 & 0.63 \\
14 EISJ2241-3932 & 61.7 & 38 & 22 & 0.55 & 0.58 \\
15 EISJ2243-4010A & 82.8 & 45 & 21 & 0.40 & 0.47 \\
16 EISJ2243-3952 & 97.5 & 49 & 44 & 0.41 & 0.90 \\
17 EISJ2243-3959 & 91.1 & 47 & 43 & 0.47 & 0.91 \\
18 EISJ2243-4010B & 93.9 & 46 & 26 & 0.38 & 0.57 \\
19 EISJ2243-3947 & 65.3 & 37 & 35 & 0.51 & 0.95 \\
20 EISJ2244-4008 & 65.6 & 41 & 35 & 0.63 & 0.85 \\
21 EISJ2244-4019 & 88.1 & 51 & 40 & 0.49 & 0.78 \\
22 EISJ2246-4012B & 54.8 & 17 & 16 & 0.27 & 0.94 \\
23 EISJ2248-4015 & 61.3 & 42 & 36 & 0.61 & 0.86 \\
\hline & & & & &
\end{tabular}

redshifts were measured by Fourier cross-correlating our spectra with standard galaxy spectra templates from Kinney et al. (1996). However, the redshift estimated by the cross-correlation was only accepted if a prominent spectral feature could be identified at a redshift consistent with that estimated by the crosscorrelation. In Paper III the accuracy of the measured redshifts was estimated to be $\sigma_{z}=0.0005$. Since the typical magnitudes are similar we adopt the same value here.

For all target clusters two slit masks were produced regardless of the local galaxy density. Therefore, we do not reach the same level of completeness (defined as the fraction of targeted galaxies in a field) in all fields. Also the efficiency for obtaining redshifts (the fraction of obtained spectra that yielded a redshift measurement) varies, mainly due to varying observing conditions. In Table 2 we summarise the results of the spectroscopic campaign. The table lists in Col. 1 the field identifier; Col. 2 the field name; in Col. 3 the area in square arcmin containing the target galaxies; in Col. 4 the number of target galaxies; in Col. 5 the number of derived redshifts; in Col. 6 the overall completeness, as defined below; in Col. 7 the overall efficiency of obtaining redshifts (the ratio between Cols. 5 and 4 ). The overall completeness given in Col. 6 is defined as the ratio of observed to all galaxies brighter than $I=20.0$ within a rectangular region. The latter is defined as the smallest rectangle covering all observed galaxies and is outlined by dashed lines in Figs. 4 and 5.

In Fig. 1 we show the distributions of the overall completeness and overall efficiency. We find that in general the overall completeness is $\sim 50 \%$. For one field (EISJ2246-4010B, \#22) only one mask was observed yielding a low completeness of only $27 \%$. The distribution of overall efficiencies show two peaks, corresponding to fields that were observed under good
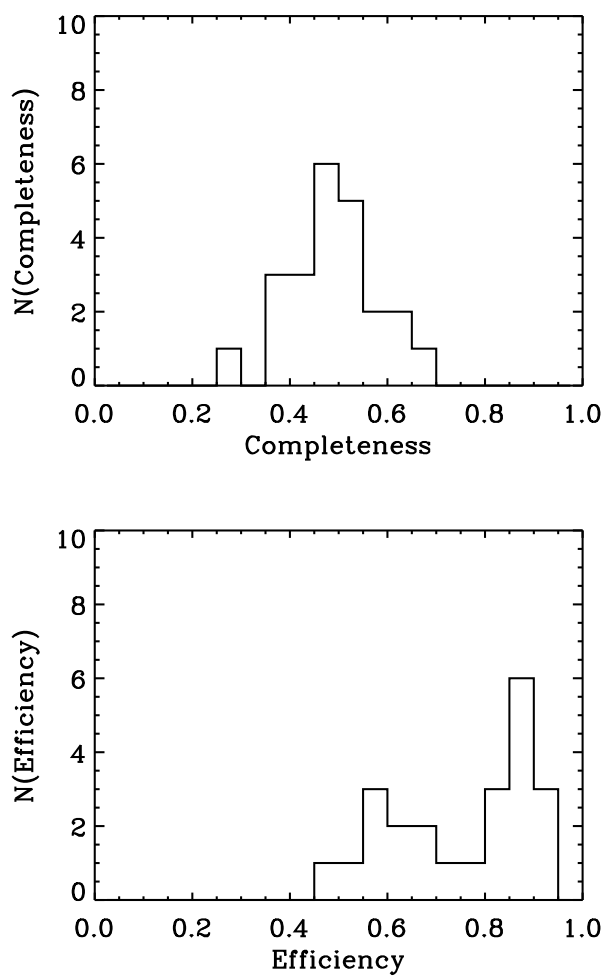

Fig. 1. The distribution of overall completeness, the fraction of targeted galaxies to all galaxies (upper panel), and overall efficiency, the fraction of spectra that yielded a redshift determination (lower panel) per field. The overall completeness is defined for galaxies brighter than $I=20$.

conditions and fields observed in less than optimal conditions due to clouds or the presence of the moon. The two peaks are centred around $\sim 60 \%$ and $\sim 85 \%$.

In Fig. 2 we show two examples of completeness (upper panel) and efficiency (lower panel) as a function of magnitude. The two examples are typical for systems with low (EISJ22364026, \#7, dashed line) and high efficiency (EISJ0049-2920, \#5, dotted line), respectively. It can be seen, from the upper panel of the figure, that the completeness is very high at the brightest magnitudes but decreases to $\sim 25 \%$ at about $I=19.75$. The efficiency in measuring redshifts, shown in the lower panel, is quite high, reaching $\sim 50 \%$ at $I \sim 20.0$ for the systems observed in good conditions. Unfortunately, the observing conditions were not homogeneous and for some systems the $50 \%$ efficiency is reached at much brighter magnitudes $(I \sim 18.5)$. From this it is clear that for some systems we are not able to obtain redshifts for galaxies with luminosities close to $L^{*}$, corresponding to the magnitude range 18.5-19.3 in the $I$-band. Therefore, for these systems the confirmation is questionable.

The variation of the effective magnitude limit of our observations from field-to-field can introduce a strong bias in our results. This can happen in two ways: one by severely undersampling higher $z$ clusters and not being able to confirm them, biasing the sample to lower redshifts; the other, similar to the first but leading to the misidentification of the more distant clusters associating them to foreground structures, and biasing our conclusions regarding the reliability of the redshift and richness estimates. We use the ratio of faint to bright galaxies around $M^{*}$ as a measure of how we probe the luminosity function at the redshift estimated for the cluster candidate. The bright galaxies are defined to be in the magnitude interval $\left[I^{*}-1: I^{*}\right]$ and faint in $\left[I^{*}: I^{*}+1\right]$. 

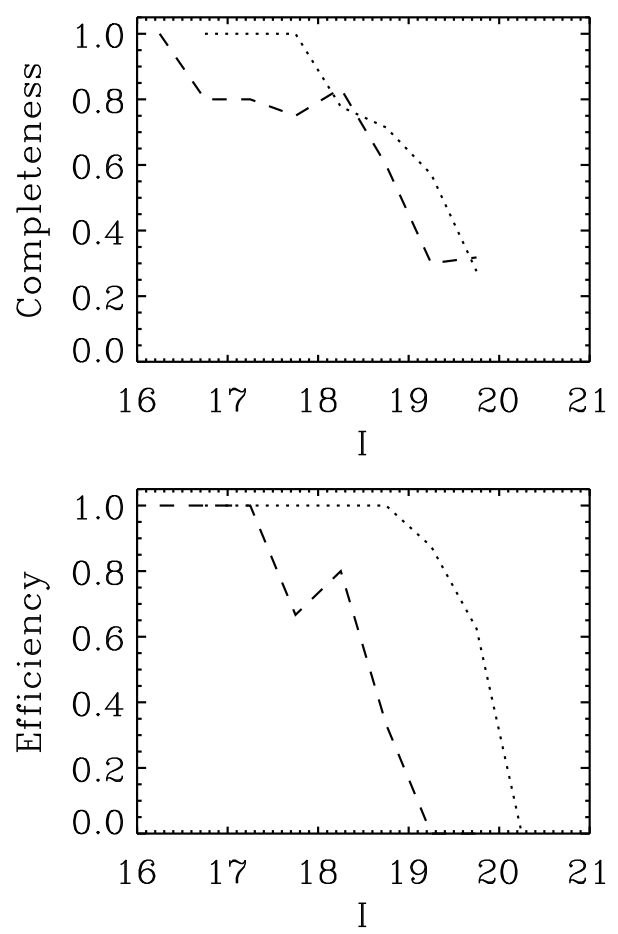

Fig. 2. The completeness (upper panel) and efficiency (lower panel) as function of magnitude as found for the clusters EISJ0049-2920 (\#5, dotted line), a typical case of high efficiency, and EISJ2236-4026 (\#7, dashed line), a typical case of low efficiency.

Table 3. Redshifts measured for the individual galaxies. Here we give the first five lines of the table. The entire table is available at CDS.

\begin{tabular}{ccccc}
\hline \hline & $\alpha(\mathrm{J} 2000)$ & $\delta(\mathrm{J} 2000)$ & $I$ & $z$ \\
\hline 1 & 11.126234 & -29.822523 & 17.89 & 0.2576 \\
2 & 11.136551 & -29.839476 & 19.90 & $0.5082:$ \\
3 & 11.153917 & -29.852741 & 18.31 & 0.2572 \\
4 & 11.156263 & -29.831465 & 18.80 & 0.1738 \\
5 & 11.187512 & -29.898016 & 18.97 & 0.6706 \\
\hline
\end{tabular}

\section{Spectroscopic results}

We measured a total of 747 redshifts for galaxies in the 23 EIS cluster fields considered, with the number of derived redshifts ranging between 16 and 45 per field. Table 3, available at the CDS, lists in Col. 1 an identifier for each galaxy; in Cols. 2 and 3 the right ascension and declination in J2000 for the galaxy; Col. 4 the I-magnitude from the EIS object catalogues (Nonino et al. 1999; Prandoni et al. 1999) and in Col. 5 the measured redshift. A colon (":") indicates that the redshift was assigned exclusively based on the cross-correlation without any recognisable spectroscopic features. An " $e$ " indicates that emission lines are present in the spectrum.

In an attempt to increase the number of redshifts in each field we searched the NASA Extragalactic Database (NED). For several fields we found no new redshifts. In others we found no more than five but, in general, these either were in common with our observations or lay at the outskirts of the field having no bearing on the confirmation of the candidate clusters. In only two cases we find one redshift for a galaxy in the central part of the fields. These cases are EISJ2246-4012B (\#22) and EISJ2248-4015 (\#23). None of these had an impact on the conclusions drawn based on our own observations, and are therefore not included in the analysis below.

\subsection{Spectroscopic confirmation of systems}

The measured redshifts were used to search for significant groups in redshift space to identify the physical systems associated to the detections by the matched-filter algorithm. As described in previous papers (Papers I, II and III) we identify groups in redshift space by the "gap"-technique of Katgert et al. (1996) with a gap-size of $\Delta z=0.005(1+z)$ corresponding to $1500 \mathrm{~km} \mathrm{~s}^{-1}$ in the restframe. The identified groups are shown as the solid histograms. For assessing the significance of the identified groups we use the CNOC2 $0223+00$ catalogue (Yee et al. 2000). As detailed in Paper II, the significance, $\sigma_{1}$, is determined from the probability of finding a group with the same number of objects or more at the same redshift imposing the same magnitude limit as for the survey. This procedure is a simple way to take into account the selection function of the present survey and the clustering of field galaxies. More advanced applications of such approaches have been presented by for example Holden et al. (1999), Ramella et al. (2000) and Gilbank et al. (2004).

To give an overview of the redshifts and their clustering, Fig. 3 shows the redshift distribution for each field. The upper parts show the bar diagram of the redshifts while the lower parts give the redshift histogram with a bin size of $\Delta z=0.01$. We find a total of 81 groups in redshift space with a least 3 members.

In Table 4 we list groups with at least three members and a significance $\sigma_{1} \geq 99 \%$ identified in each cluster field. The remaining less significant detections are considered marginal and given in Table A.1. The tables list in Cols. 1 and 2 the field identifier and the cluster field name; in Col. 3 the number of spectroscopic members of the group; in Cols. 4 and 5 the mean position in J2000; in Col. 6 the mean redshift of the group members; in Col. 7 the velocity dispersion corrected for our measurement accuracy. In cases where the measured velocity dispersion is smaller than the measurement error we list the value of $\sigma_{v}=0$; in Col. 8 the significance as defined above and in Col. 9 the distance in arcmin between the group and the original MF position.

The table lists 35 significant groups, ranging between zero and four groups per cluster field, having from three to $25 \mathrm{mem}-$ bers. In four fields no significant groups were identified. As in Paper III the group associated with a matched filter detection is chosen as follows: 1) The richest group in the field, if it has a significantly larger number of members than the other groups; 2) The one closest to the EIS position, if two groups have roughly the same number of members; 3) The most concentrated group, if two groups are close to the EIS position and have almost the same number of members. To help in the analysis the images and the spatial distribution of the galaxies are shown in Figs. 4 and 5. The figures show the central image cutout with $\sim 4^{\prime}$ on the side and the spatial distributions of the galaxies within the surveyed area. The projected distribution includes all galaxies with $I \leq 20.0$ in the cluster regions. In the figures symbols indicate galaxies with measured redshift associated (solid symbols) or not (crosses) to a group. In Fig. 4 only the group associated to the EIS detection is marked. Note that small and large symbols are shown in four cases. This is because examination of the redshift distribution of these groups suggests the presence of substructures. The circle has a radius of $0.5 h_{75}^{-1} \mathrm{Mpc}$ at the redshift of the confirmed group and is centred at the original position of the matched filter detection.

From the analysis we find that in 19 of the 23 fields there are between one and four significant overdensities in redshift space. 

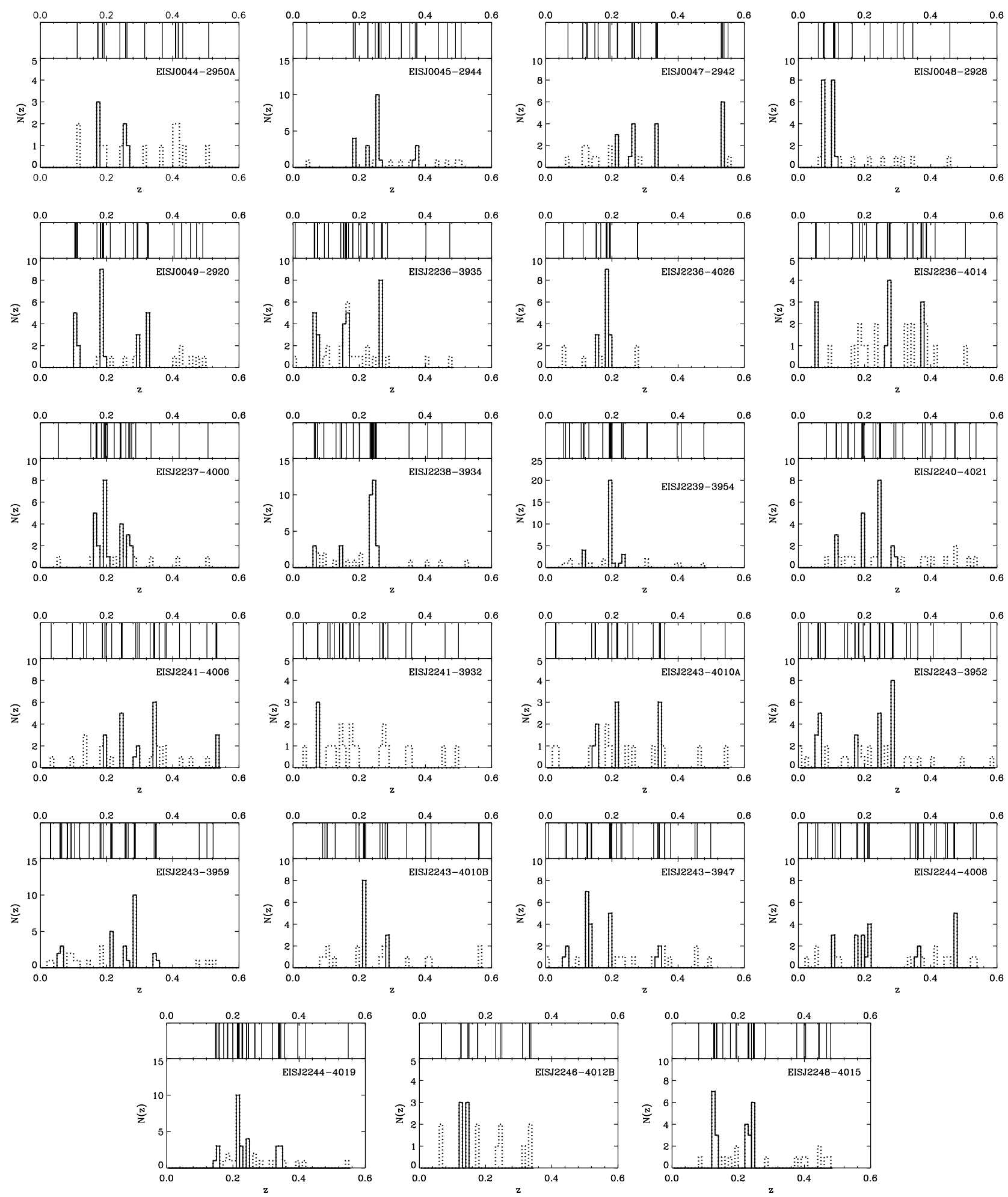

Fig. 3. Redshift distributions for the observed cluster fields as indicated in each panel. Note that the scale of the $y$-axis differs between the panels. The upper panels show bar diagrams of the measured redshifts, while the lower panels give the corresponding histograms of the redshift distributions (dashed line). The solid lines mark the detected groups.

In 10 fields (43\% of the entire set) we assigned one of the significant detections to the EIS cluster. These detections are marked in bold face in Table 4. In each field the assigned group turned out to be the one closest to the original position with distances ranging between $0.1^{\prime}$ and $1.9^{\prime}$. It can be seen that most of the assigned groups are found within $1^{\prime}$ corresponding to a few core radii at the redshifts discussed here. For the other groups the distance to the original position is in general much larger than this. All, except one, of the 10 confirming systems have at least six members located within a radius of $0.5 h_{75}^{-1} \mathrm{Mpc}$. Whether these systems are bound or not can only be properly assessed by much denser spectroscopic observations. However, Gal et al. (2008) 

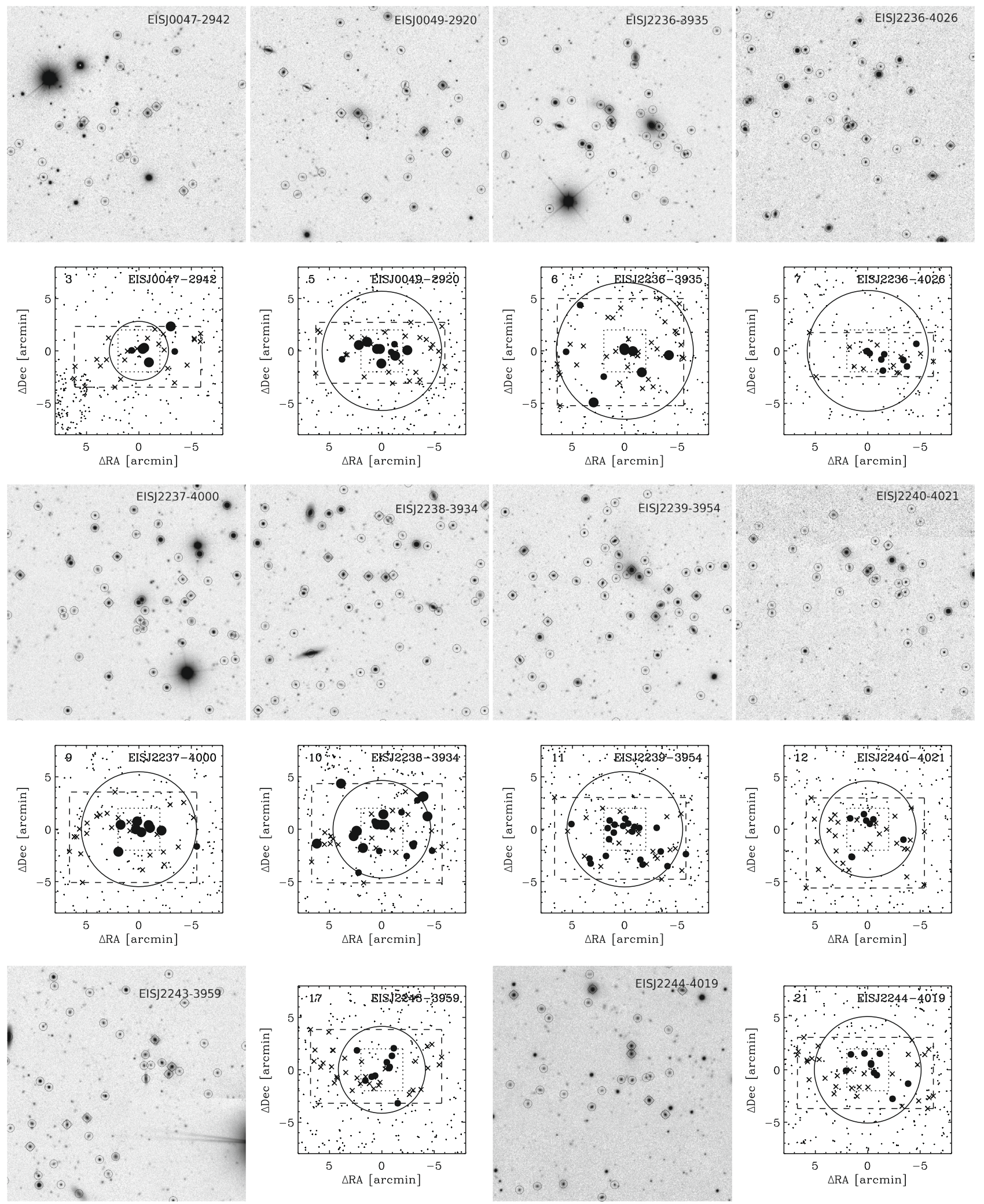

Fig. 4. Image cutouts, centred at the original MF position and with a size of $\sim 4^{\prime}$, and position plots for the ten fields with groups assigned to the EIS detection. In the image cutouts galaxies with $I<21$ are marked by circles and galaxies with redshifts and belonging to the group are indicated by diamonds. In the projected distributions all galaxies with $I \leq 20$ are indicated by the small dots, crosses mark galaxies with redshift and the member galaxies are marked by filled circles. The large circles centred on the MF position indicate a radius of $0.5 h_{75}^{-1} \mathrm{Mpc}$. In cases where there are symbols of different size it relates to substructures in the redshift distribution. The dashed rectangle marks the region of the redshift survey and the dotted square outlines the area of the image cutout. 
L. F. Grove et al.: Multi-object spectroscopy of low-redshift EIS clusters. IV.
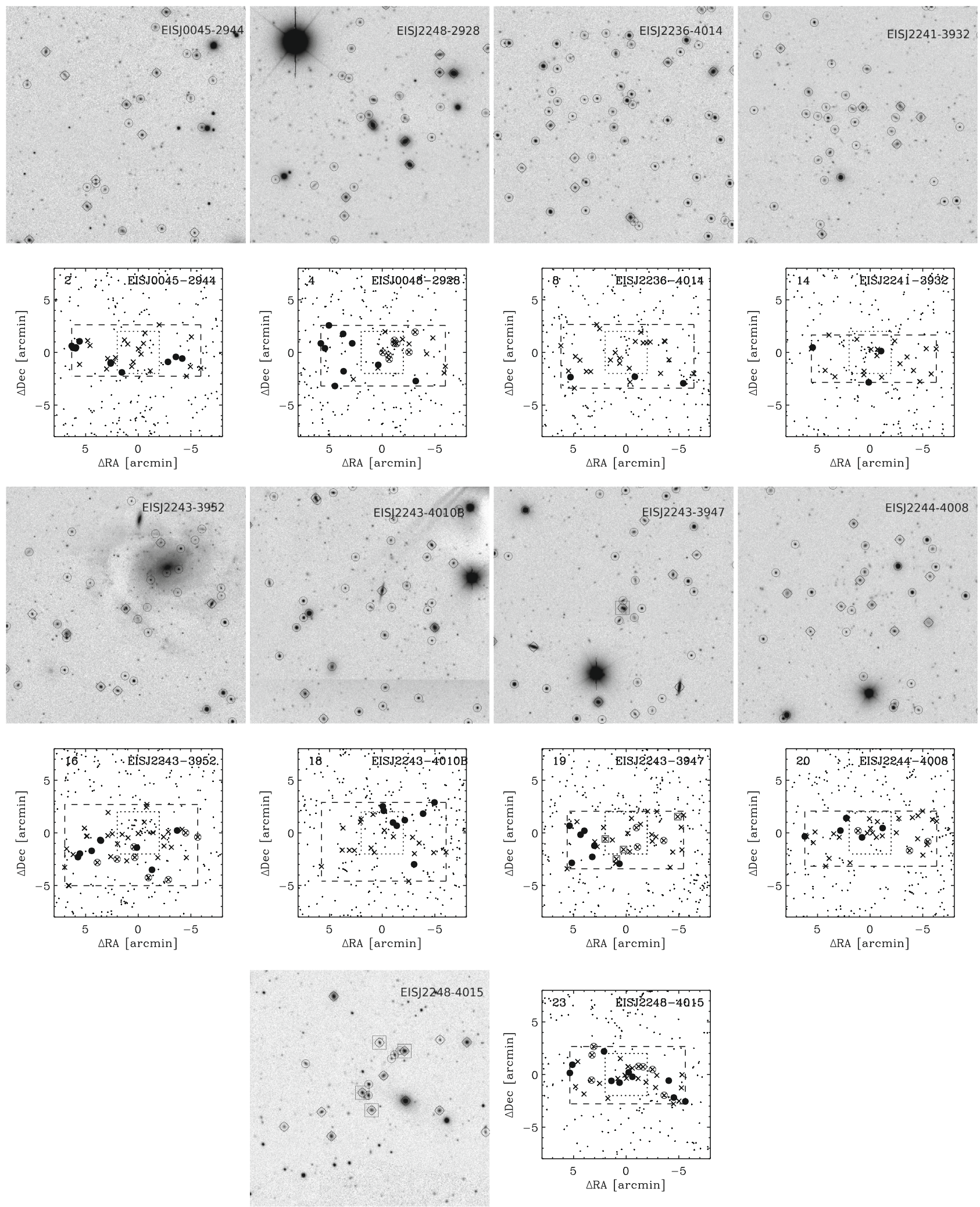

Fig. 5. Image cutouts, centred at the original MF position and with a size of $\sim 4^{\prime}$, and position plots for the nine fields with significant groups detected but not assigned to the EIS cluster candidate. In the image cutouts galaxies with $I<21$ are marked by circles and galaxies with redshifts are indicated by diamonds. In the field of EISJ2243-3942 the galaxy marked by the big square has a redshift of 0.2301. For EISJ2248-4015 the big squares mark four galaxies with redshifts in the range $0.2460-0.2484$. In the projected distributions all galaxies with $I \leq 20$ are indicated by the small dots, crosses mark galaxies with redshift and each significant group is denoted by its own symbol. The dashed rectangle marks the region of the redshift survey and the dotted square outlines the area of the image cutout. 
Table 4. Identified groups with a significance of at least $99 \%$. The ones in bold face are the ones we interpret as related to the cluster detection.

\begin{tabular}{|c|c|c|c|c|c|c|c|c|}
\hline ID & Cluster field & Members & $\alpha(\mathrm{J} 2000)$ & $\delta(\mathrm{J} 2000)$ & $z$ & $\sigma_{v}\left[\mathrm{~km} \mathrm{~s}^{-1}\right]^{a}$ & $\sigma_{1}[\%]$ & Dist. ['] \\
\hline 1 & EISJ0044-2950A ${ }^{b}$ & - & - & - & - & - & - & - \\
\hline 2 & EISJ0045-2944 & 11 & 004511.8 & -294513.1 & 0.259 & 424 & 99.9 & 2.4 \\
\hline 3 & EISJ0047-2942 & 6 & 004717.2 & -294241.9 & 0.534 & 418 & 99.9 & 1.3 \\
\hline 4 & EISJ0048-2928 3 & 8 & 004819.7 & -292820.9 & 0.077 & 111 & 99.9 & 1.4 \\
\hline 4 & EISJ0048-2928 & 9 & 004840.3 & -29 2906.2 & 0.108 & 289 & 99.9 & 2.0 \\
\hline 5 & EISJ0049-2920 & 7 & 004926.8 & -292204.9 & 0.108 & 773 & 99.9 & 1.8 \\
\hline 5 & EISJ0049-2920 & 10 & 004932.3 & -292034.7 & 0.187 & 781 & 99.9 & 0.2 \\
\hline 6 & EISJ2236-3935 & 5 & 223620.3 & -393530.6 & 0.065 & 161 & 99.9 & 3.3 \\
\hline 6 & EISJ2236-3935 & 3 & 223551.8 & -393603.5 & 0.074 & 0 & 99.9 & 2.2 \\
\hline 6 & EISJ2236-3935 & 9 & 223607.7 & -393608.7 & 0.158 & 915 & 99.9 & 1.1 \\
\hline 6 & EISJ2236-3935 & 8 & 223556.2 & -393508.0 & 0.269 & 125 & 99.9 & 1.4 \\
\hline 7 & EISJ2236-4026 & 9 & 223638.1 & -402650.8 & 0.184 & 151 & 99.9 & 1.9 \\
\hline 8 & EISJ2236-4014 & 3 & 223650.9 & -401724.6 & 0.053 & 295 & 99.9 & 2.5 \\
\hline 9 & EISJ2237-4000 & 7 & 223719.8 & -395938.5 & 0.169 & 239 & 99.9 & 1.7 \\
\hline 9 & EISJ2237-4000 & 9 & 223708.1 & -400032.9 & 0.196 & 604 & 99.9 & 0.7 \\
\hline 10 & EISJ2238-3934 & 3 & 223816.8 & -393557.0 & 0.065 & 489 & 99.9 & 2.8 \\
\hline 10 & EISJ2238-3934 & 25 & 223801.6 & -393449.7 & 0.243 & 1103 & 99.9 & 0.3 \\
\hline 11 & EISJ2239-3954 & 4 & 223922.5 & -395738.2 & 0.115 & 0 & 99.2 & 3.2 \\
\hline 11 & EISJ2239-3954 & 21 & 223917.8 & -395532.8 & 0.195 & 420 & 99.9 & 1.0 \\
\hline 12 & EISJ2240-4021 & 5 & 224012.6 & -402218.5 & 0.195 & 545 & 99.0 & 1.5 \\
\hline 12 & EISJ2240-4021 & 8 & 224008.5 & -402119.5 & 0.247 & 199 & 99.6 & 0.2 \\
\hline 13 & EISJ2241-4006 ${ }^{b}$ & - & - & - & - & - & - & - \\
\hline 14 & EISJ2241-3932 ${ }^{c}$ & 3 & 224139.2 & -393255.2 & 0.074 & 102 & 99.9 & 1.7 \\
\hline 15 & EISJ2243-4010A $\mathrm{A}^{b, c}$ & - & - & - & - & - & - & - \\
\hline 16 & EISJ2243-3952 & 8 & 224314.6 & -395456.7 & 0.062 & 723 & 99.9 & 2.4 \\
\hline 16 & EISJ2243-3952 & 8 & 224331.1 & -39 5411.8 & 0.285 & 33 & 99.2 & 2.7 \\
\hline 17 & EISJ2243-3959 & 5 & 224333.4 & -395746.8 & 0.061 & 680 & 99.9 & 1.9 \\
\hline 17 & EISJ2243-3959 & 10 & 224329.4 & -395927.8 & 0.285 & 117 & 99.8 & 0.1 \\
\hline 18 & EISJ2243-4010B & 8 & 224331.8 & -400921.3 & 0.215 & 457 & 99.9 & 2.4 \\
\hline 19 & EISJ2243-3947 & 3 & 224346.3 & -394800.3 & 0.062 & 677 & 99.9 & 1.9 \\
\hline 19 & EISJ2243-3947 & 7 & 224415.2 & -394842.5 & 0.126 & 176 & 99.9 & 3.9 \\
\hline 19 & EISJ2243-3947 & 4 & 224353.8 & -39 4815.3 & 0.138 & 53 & 99.3 & 0.9 \\
\hline 20 & EISJ2244-4008 & 3 & 224407.7 & -400908.1 & 0.102 & 0 & 99.8 & 2.8 \\
\hline 20 & EISJ2244-4008 & 5 & 224433.2 & -400805.7 & 0.471 & 63 & 99.6 & 2.2 \\
\hline 21 & EISJ2244-4019 & 4 & 224416.3 & -401953.7 & 0.153 & 1210 & 99.0 & 2.3 \\
\hline 21 & EISJ2244-4019 & 10 & 224425.5 & -401942.2 & 0.216 & 325 & 99.9 & 0.6 \\
\hline 22 & EISJ2246-4012B ${ }^{b, c}$ & - & - & - & - & - & - & - \\
\hline 23 & EISJ2248-4015 & 10 & 224854.6 & -401539.2 & 0.129 & 863 & 99.9 & 0.3 \\
\hline 23 & EISJ2248-4015 & 7 & 224855.3 & -401444.4 & 0.230 & 176 & 99.2 & 0.6 \\
\hline
\end{tabular}

${ }^{a} \sigma_{v}=0$ reflects a measured velocity dispersion below the measurement error; ${ }^{b}$ no significant groups were detected in this field.

found that with this search radius, it is common to find un-bound systems with up to four members, but not six. Therefore, we consider it likely, but not conclusive, that the assigned groups represent bound systems. As discussed below, we should point out that close inspection of the redshift distribution of one of the fields (EISJ2238-3934, \#10) led us to split this system into two. Another system worth mentioning is EISJ2236-4026 (\#7) that was associated to a lower redshift group despite the poor sampling of the luminosity function as indicated by the ratio of faint to bright galaxies, and the lack of measured redshifts at its originally estimated redshift. This association was based on the visual inspection of the image, which does not show sign of a concentration of fainter galaxies. Finally, note that due to the poor sampling of the luminosity function at $z \geq 0.4$ only one system was confirmed at this redshift from the present data, demonstrating the intrinsic bias of our results.

For nine fields, significant groups are identified but not assigned to an EIS detection, even though in some cases the redshift is comparable to the matched-filter estimates. This is because they fail in some other respect. For instance, in some cases, we discard a group as the true confirmation due to poor coverage of the central regions or strong evidence of a concentration 


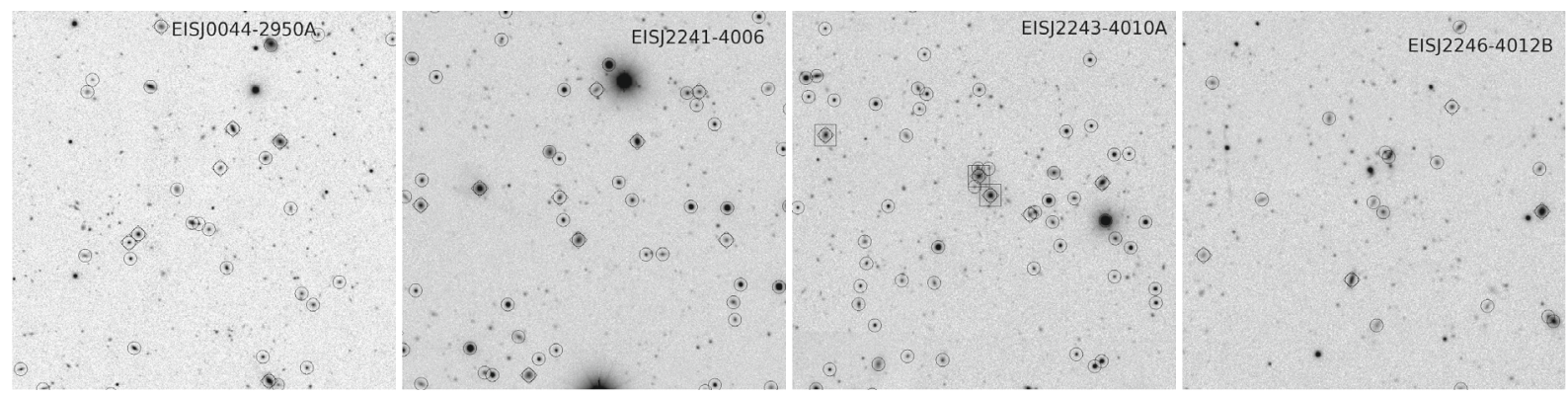

Fig. 6. Image cutouts for the four fields without significant groups detected. From left to right the panels show the field of EISJ0044-2950A, EISJ2241-4006, EISJ2243-4010A and EISJ2246-4012B. The cutouts are centred at the original position and have a size of $\sim 4^{\prime}$. Galaxies with $I<21$ are marked by circles, additional diamonds denote galaxies with measured redshifts. In the field of EISJ2243-4010A the galaxies marked by the big squares have redshifts in the range $0.3431-0.3471$.

of fainter galaxies at the matched-filter candidate position. The analysis shows that for one cluster (EISJ2248-4015, \#23) a marginal detection with a significance of $98.7 \%$ is likely the origin of the detection. A detailed account for each group is presented in Appendix B.

Finally, for the remaining four fields, no significant group was detected. The image cutouts of these fields are shown in Fig. 6. From the images it can be seen that for the clusters EISJ0044-2950 (\#1) and EISJ2241-4006 (\#13) there is little indication of a concentration of galaxies. The origin of the matched filter detection is in these cases unclear. In the field of EISJ2243-4010A a compact group of galaxies dominated by two bright galaxies is found. The two bright galaxies have very similar redshifts consistent with that originally estimated for the cluster $\left(z_{\mathrm{MF}}=0.3\right)$. This is suggestive of a group of galaxies but in redshift space the group only has a significance of $73.4 \%$ (listed in Table A.1), thus is not part of the sample considered significant. Additional spectroscopy of the region may confirm the existence of a group of galaxies. In the last field, EISJ22464012B (\#22) essentially no redshifts were obtained in the central region of the field. This field was only covered by one slit mask due to the lack of time. The weak concentration of galaxies in the centre may represent a group of galaxies. The confirmation of this requires additional spectroscopic data.

To summarise, we find 10 candidates confirmed by the present spectroscopic data. For the remaining 13 fields the available data are not sufficient to assign a significant group to the EIS detection. However, careful examination of the available data showed that in two cases a marginally significant detection in redshift space is likely the origin of the matched-filter candidate. Four systems $(\sim 20 \%)$ do not show any signs of a concentration of galaxies and the origin of the matched filter detection remains unclear. For the remaining seven cases the available data are insufficient to draw a final conclusion. Of the original sample 14 were at $z_{\mathrm{MF}}=0.3$ of which we confirm $8(\sim 57 \%)$. Of the 9 candidates with $z_{\mathrm{Mf}}=0.4$ we confirm only $2(\sim 22 \%)$.

Below we investigate in more detail the properties of the confirmed systems. Table 5 summarises these results and will be used throughout the rest of the paper. The table gives in Col. 1 the field identifier; in Col. 2 the name of the cluster field; in Col. 3 the number of spectroscopic members; in Col. 4 the matched filter redshift estimate; in Col. 5 the spectroscopic redshift; in Col. 6 the velocity dispersion with $68 \%$ bootstrap errors; in Col. 7 updated $\Lambda_{\text {cl,new }}$-richness as described below; in Cols. 8 and 9 the colour of the identified photometric red sequence and the confidence level of its detection as described in Sect. 4.2; in Cols. 10 and 11 the colour of the red sequence of the spectroscopic members and its significance (Sect. 4.2); and in Col. 12 the measured colour scatter for the spectroscopic members. The five systems without colour information are indicated by N.A. in the table.

\subsection{Reliability of the matched-filter redshifts}

For any catalogue of cluster candidates it is important to establish the reliability of the estimated redshifts, since systematic spectroscopic follow-up is, in general, not possible. Here we investigate the reliability of the redshifts estimated by the matched-filter algorithm. The redshift estimates, reported in Table 5, are obtained using the mean values for consistency with previous papers. Due to the sample sizes we have investigated the consistency of these mean values with the results of the median and biweight estimators (e.g. Beers et al. 1990). It was established that the three measures agreed to within $\delta z \sim 0.002$ (Paper III).

A summary of the comparison between the estimated and measured redshifts is presented in Fig. 7. In the upper panel we show $z_{\text {spec }}$ versus $z_{\mathrm{MF}}$ obtained for the sample of clusters presented in this paper (filled circles). In the figure we also include points from the $z_{\mathrm{MF}}=0.2$ sample of Paper III (open circles) and for completeness the results obtained for the two marginal detections discussed above (open triangles). In the lower panel we show a normalised distribution of the redshift differences, comparing that of the present sample (solid line) with that of Paper III (dashed line). The case of EISJ2236-4026 (\#7) stands out as having a large discrepancy between the estimated and measured redshift. However, its impact on the average values reported below is only marginal.

From the upper panel it is seen that, except for one system, all have measured redshifts smaller than 0.3 , yielding a mean redshift of $\left\langle z_{\text {spec }}\right\rangle=0.24 \pm 0.11$, only marginally higher than the value of $\left\langle z_{\text {spec }}\right\rangle=0.18 \pm 0.06$ obtained for the Paper III sample, and definitely not as high as one would have expected from our initial selection of systems with estimated redshifts in the range $0.3 \leq z_{\mathrm{MF}} \leq 0.4$. However, as mentioned before this is most likely a consequence of the observational bias created by the relatively bright limiting magnitude of the present spectroscopic survey. As mentioned in the last section over half of the systems in our sample have not been confirmed by the present data, with some showing clear evidence for the presence of concentrations of fainter galaxies.

In this paper we find that a mean redshift difference of $\langle\Delta z\rangle=$ $\left\langle z_{\mathrm{spec}}-z_{\mathrm{MF}}\right\rangle=-0.08 \pm 0.1$. This is consistent with the value of $\Delta z=-0.1$ estimated by Olsen (2000) from simulated data, but significantly larger than the value of $\langle\Delta z\rangle=-0.01 \pm 0.06$ obtained for the $z_{\mathrm{MF}}=0.2$ sample of Paper III. Disregarding 
Table 5. Properties of the confirmed EIS clusters and groups.

\begin{tabular}{|c|c|c|c|c|c|c|c|c|c|c|c|}
\hline Id & Cluster & \#mem & $z_{\mathrm{MF}}$ & $z_{\text {spec }}$ & $\sigma_{v}\left[\mathrm{~km} \mathrm{~s}^{-1}\right]$ & $\Lambda_{\text {cl,new }}$ & $(V-I)_{\mathrm{ph}}$ & $\sigma_{S / N}$ & $(V-I)_{\mathrm{sp}}$ & $\sigma_{\text {spec }}$ & Scatter \\
\hline 3 & EISJ0047-2942 & 6 & 0.4 & 0.534 & $617_{-473}^{+158}$ & 64.6 & - & - & - & - & - \\
\hline 5 & EISJ0049-2920 & 10 & 0.3 & 0.187 & $893_{-676}^{+266}$ & 23.9 & - & - & 1.500 & 99.8 & 0.151 \\
\hline 6 & EISJ2236-3935 & 9 & 0.3 & 0.158 & $1160_{-1004}^{+60}$ & 13.3 & N.A. & N.A. & N.A. & N.A. & N.A. \\
\hline 7 & EISJ2236-4026 & 9 & 0.4 & 0.184 & $187_{-115}^{+48}$ & 31.8 & - & - & 1.575 & 99.6 & 0.104 \\
\hline 9 & EISJ2237-4000 & 9 & 0.3 & 0.196 & $373_{-299}^{+344}$ & 11.9 & N.A. & N.A. & N.A. & N.A. & N.A. \\
\hline 10 & EISJ2238-3934 & 25 & 0.3 & 0.243 & $1433_{-167}^{+158}$ & 34.9 & N.A. & N.A. & N.A. & N.A. & N.A. \\
\hline 11 & EISJ2239-3954 & 21 & 0.3 & 0.195 & $475_{-117}^{+93}$ & 30.9 & N.A. & N.A. & N.A. & N.A. & N.A. \\
\hline 12 & EISJ2240-4021 & 8 & 0.3 & 0.247 & $268_{-148}^{+57}$ & 20.8 & 1.800 & 94.8 & - & - & - \\
\hline 17 & EISJ2243-3959 & 10 & 0.3 & 0.285 & $175_{-115}^{+41}$ & 38.1 & N.A. & N.A. & N.A. & N.A. & N.A. \\
\hline 21 & EISJ2244-4019 & 10 & 0.3 & 0.216 & $393_{-164}^{+73}$ & 22.9 & 1.500 & 96.6 & 1.425 & $>99.9$ & 0.103 \\
\hline
\end{tabular}
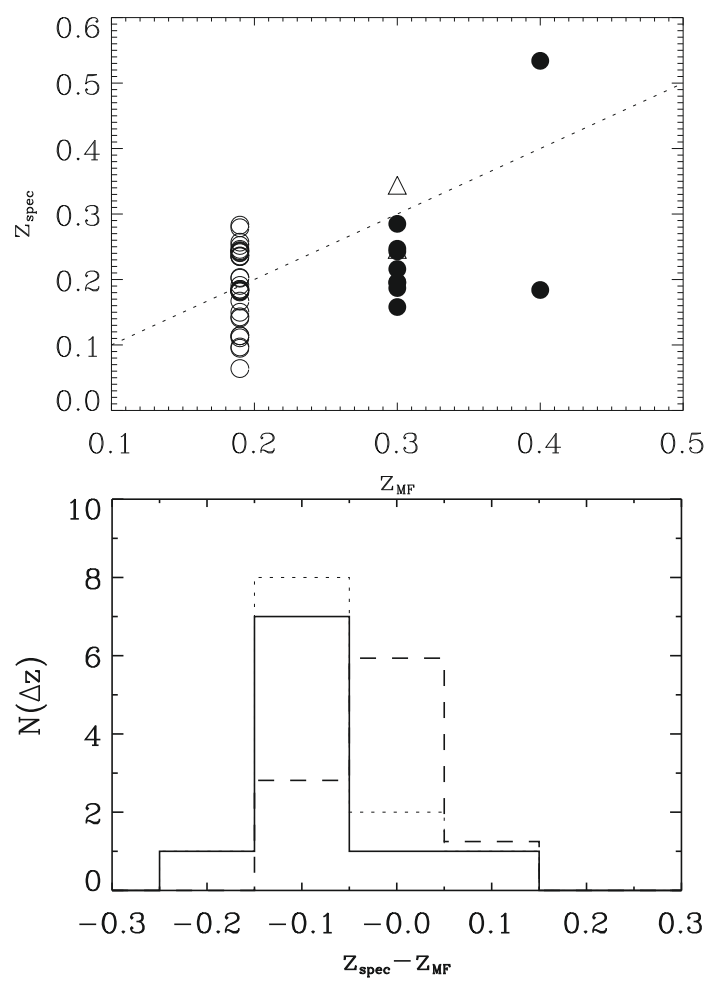

Fig. 7. Upper panel: the spectroscopic versus matched filter redshifts. Solid circles mark the confirmed systems, open circles mark systems from Paper III and triangles mark those systems that have a suggestive spectroscopic redshift. Lower panel: the distribution of the offset between the spectroscopic and matched-filter estimated redshifts of all the confirmed systems in this paper (solid line) and in Paper III (dashed line, scaled to match the same number of objects). The dotted histogram includes the two systems with a suggestive spectroscopic redshift.

EISJ2236-4026 (\#7) from consideration would change the average offset for the present sample to $\langle\Delta z\rangle=-0.06 \pm 0.08$, thus only a minor difference.

Another potential source of bias that we have considered may arise from the fact that the luminosity function of clusters of galaxies varies with the mass of the system (Zandivarez et al. 2006) with poorer systems having fainter magnitudes. If this is the case, conceivably the difference between the matched-filter and spectroscopic redshifts would vary depending on the cluster population being considered. If this effect plays a significant role we would expect to find a relation ship between the measured velocity dispersions and the redshift offsets. However, such a relation is not found in our data, so we conclude that this effect is not causing any significant bias in this work.

\subsection{Velocity dispersions}

The velocity dispersion of a galaxy cluster is a commonly used estimator of its mass and thus its distribution is expected to reflect the mass distribution of a cluster population. As discussed in Paper III different estimators (gapper, biweight and standard deviation) have been tested to determine how sensitive the values of the velocity dispersion are when considering samples that have a small number of members with measured redshifts and possible substructures as observed in the redshift distributions shown in Fig. 8. As before, we found that the different estimators yield consistent values, differing by no more than $50 \mathrm{~km} \mathrm{~s}^{-1}$. In Table 5 we use the biweight estimator found to be more robust in the presence of outliers. The velocity dispersions are rest-frame values corrected for our estimated redshift accuracy. The estimates with $68 \%$ bootstrap errors are given in Table 5 . We note that the values listed in Table 5 may differ from those in Table 4 because a different estimator was used.

The distribution of velocity dispersions is shown in Fig. 9 for the systems identified in this paper (solid histograms). In the figure we also show the same distribution for the $z_{\mathrm{MF}}=0.2$ sample (dashed histogram) of Paper III, normalised to the same number of systems. Given the small number of systems in the two samples the two distributions are very similar. Moreover, as discussed in the previous paper, the observed distributions are comparable to that of Abell clusters (Fig. 7 of Paper III) with, perhaps, a larger fraction of low velocity dispersion systems. The measured velocity dispersions cover a broad range of values, comparable to that recently reported for the SDSS cluster sample (Becker et al. 2007), varying from groups $\left(\sim 200 \mathrm{~km} \mathrm{~s}^{-1}\right)$ to rich clusters $\left(\sim 800 \mathrm{~km} \mathrm{~s}^{-1}\right)$. There are, however, some systems with large velocity dispersions such as EISJ2236-3935 (\#6) and EISJ2238-3934 (\#10), with $1160 \mathrm{~km} \mathrm{~s}^{-1}$ and $1433 \mathrm{~km} \mathrm{~s}^{-1}$ suggestive of very massive systems, unlikely to be detected within the small volumes probed by our survey. Examination of Fig. 8 suggests that in at least five cases (\#3, \#5, \#6, \#9, \#10), or $50 \%$ of the confirmed systems, the presence of peaks in the redshift distribution along the line of sight of these systems, separated by $\sim 2000 \mathrm{~km} \mathrm{~s}^{-1}$, are clearly visible and may influence our estimate of the velocity dispersion. This may reflect the presence of interlopers or possibly bound infalling systems frequently observed (e.g. Buote \& Tsai 1996). The presence of these interlopers may bias the velocity dispersion estimates, especially in the case of \#6 and \#10. While the sample considered here is small, 

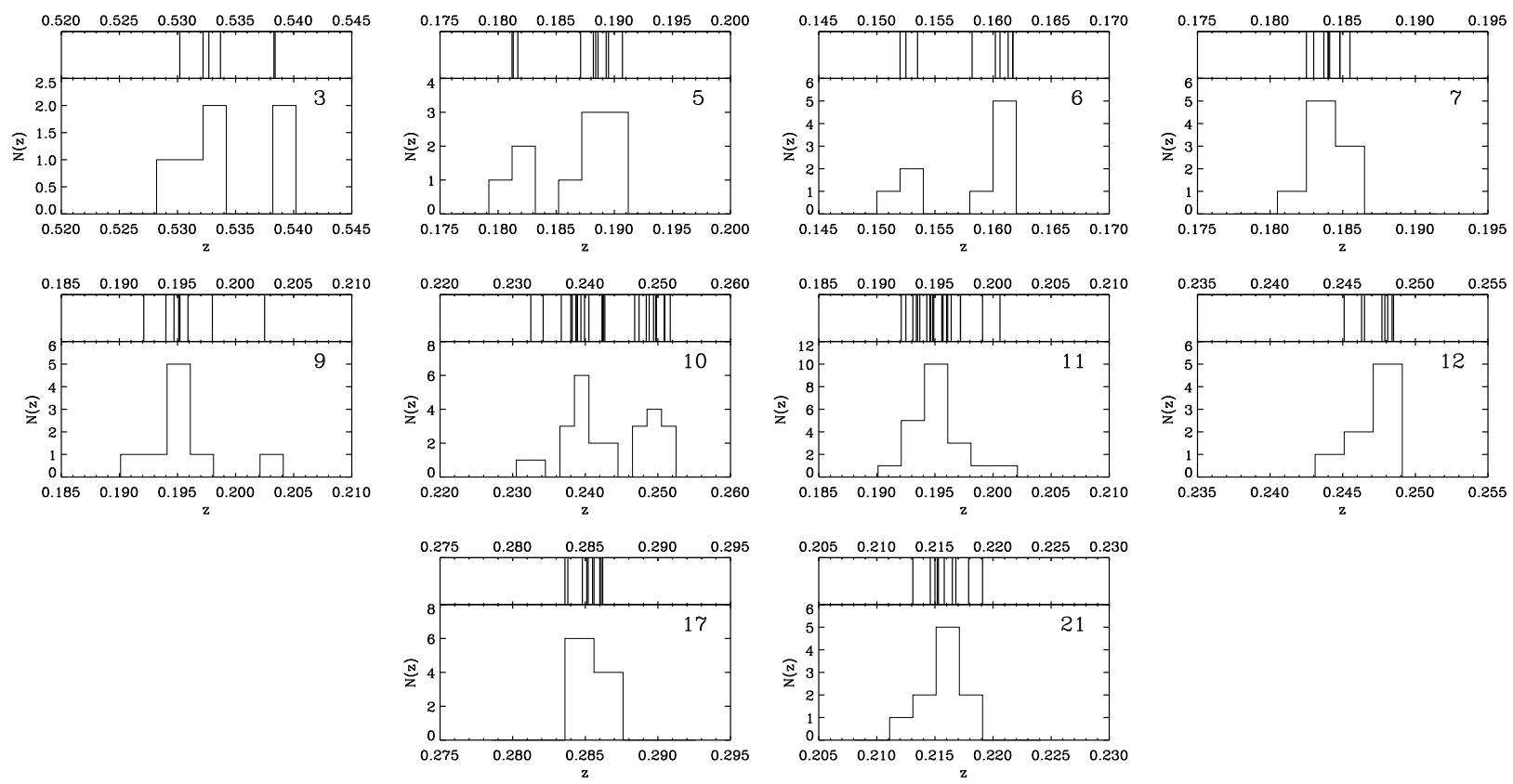

Fig. 8. Detailed redshift distributions for all systems considered confirmed.

it is worth noting the high frequency of such cases, even though comparable with other recent findings (Jeltema et al. 2008).

In order to investigate the nature of the interlopers, we have examined the spatial distribution of these systems and the relative position of the galaxies found in the different subgroups. In Fig. 4 galaxies belonging to different subgroups are indicated by the size of the symbols. From a close examination of Figs. 8 and 4 we find that: 1) EISJ0047-2942 (\#3) consists of one dominant group twice as large as the secondary peak located in the background, which we consider to be unrelated to the main cluster. Therefore we keep only the dominant group; 2) EISJ0049-2920 (\#5) is similar to the previous case and we keep only the dominant group which in this case is the most distant one; 3) EISJ2236-3935(\#6) is similar to the previous case and we keep only the group at the highest redshift, considerably decreasing our measured velocity dispersion; 4) EISJ22374000 (\#9) is the easiest case with a dominant group with a single interloper at a higher redshift which can be neglected without significantly impacting the value calculated for the velocity dispersion as can be seen by comparing the two reported values; 5) EISJ2238-3934 (\#10) with the largest velocity dispersion consists of two comparable clumps, one with 13 members and the other with 10, and one foreground group composed of only two galaxies, which we consider as field interlopers and exclude from further analysis. Therefore, we consider these as two independent clusters.

Based on the above considerations we recomputed the velocity dispersion considering only the richest group in the systems \#3, \#5 and \#6, removing the interloper in \#9 and splitting the cluster \#10 into two. The results are summarised in Table 6 which lists in Col. 1 the field identifier; in Col. 2 the name of the cluster field; in Col. 3 the number of spectroscopic members; in Col. 4 the matched filter redshift estimate; in Col. 5 the spectroscopic redshift; and in Col. 6 the velocity dispersion with $68 \%$ bootstrap errors. The table shows that the five systems are now represented by six clusters of which five have significantly smaller velocity dispersions than the original detections, adding four new systems to the velocity dispersions range $250 \mathrm{~km} \mathrm{~s}^{-1}$ to
$500 \mathrm{~km} \mathrm{~s}^{-1}$ and one with velocity dispersion $<250 \mathrm{~km} \mathrm{~s}^{-1}$. This new distribution is also shown in Fig. 9 (dotted histogram) which shows a high concentration of systems close to the peak value of $\sim 400 \mathrm{~km} \mathrm{~s}^{-1}$ found for the much larger sample by Becker et al. (2007). Our sample is arguably small making the above discussion very subjective. However, only a much better sampling of each system would allow to fully characterise the nature of the systems identified in redshift space.

\section{Photometric properties of the detected systems}

In addition to the spectroscopic results reported above, the photometric properties of the confirmed systems are also important for comparison with other cluster samples possibly having other selection effects. In this section we will further characterise the 10 confirmed clusters in terms of their richness and photometric properties of their galaxy population as well as compare these properties with those of the $z_{\mathrm{MF}}=0.2$ sample discussed in Paper III.

\subsection{Richness}

The matched filter algorithm provides a measure of the richness $\left(\Lambda_{\mathrm{cl}}\right)$ for the cluster candidates based on the estimated redshifts, where the $\Lambda_{\mathrm{cl}}$-richness is the number of $L^{*}$-galaxies required to match the total luminosity of the cluster. Computation of the richness depends on the apparent Schechter magnitude and angular extent of the cluster, which at these redshifts vary rapidly. Therefore, even though the spectroscopic and estimated redshifts are in reasonable agreement we have to recompute the cluster richness using the assigned spectroscopic redshift and the centre of the assigned groups as listed in Table 4 . The new richness values $\left(\Lambda_{\mathrm{cl}, \text { new }}\right)$ are listed in Table 5. In general, since the matchedfilter tends to overestimate the redshifts it also overestimates the richness. Note, however, that for EISJ0047-2942 (\#3) the opposite is true with the corrected richness being much larger than the 
Table 6. Updated properties of the groups showing substructure.

\begin{tabular}{rlrrrrrr}
\hline \hline Id & Cluster & $\alpha(\mathrm{J} 2000)$ & $\delta(\mathrm{J} 2000)$ & \#mem & $z_{\mathrm{MF}}$ & $z_{\text {spec }}$ & $\sigma_{v}\left[\mathrm{~km} \mathrm{~s}^{-1}\right.$ \\
\hline 3 & EISJ0047-2942 & $00: 47: 17.4$ & $-29: 42: 33$ & 4 & 0.4 & 0.532 & $251_{-101}^{+109}$ \\
5 & EISJ0049-2920 & $00: 49: 31.7$ & $-29: 20: 33$ & 7 & 0.3 & 0.189 & $248_{-112}^{+116}$ \\
6 & EISJ2236-3935 & $22: 35: 59.9$ & $-39: 36: 45$ & 6 & 0.3 & 0.161 & $284_{-169}^{+258}$ \\
9 & EISJ2237-4000 & $22: 37: 11.3$ & $-40: 00: 23$ & 8 & 0.3 & 0.195 & $350_{-171}^{+225}$ \\
10 & EISJ2238-3934 & $22: 38: 06.3$ & $-39: 34: 03$ & 13 & 0.3 & 0.240 & $497_{-148}^{+55}$ \\
10 & EISJ2238-3934 & $22: 37: 54.0$ & $-39: 35: 51$ & 10 & 0.3 & 0.249 & $357_{-99}^{+55}$ \\
\hline
\end{tabular}

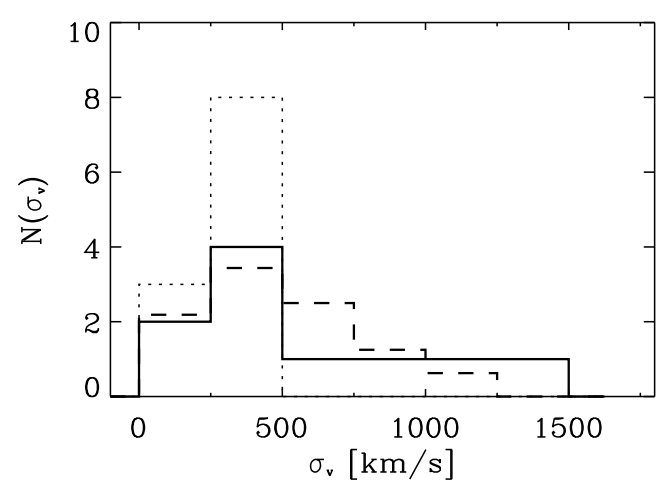

Fig. 9. Distribution of velocity dispersions (solid line) compared with that of the $z_{\mathrm{MF}}=0.2$ sample scaled to the same number of objects (dashed line, Paper III). The dotted histogram denote the distribution assuming that the four systems with substructure are superpositions and only the richest group is included as the confirmation.

original estimate. This is because the redshift was significantly underestimated.

A large scatter of the relation between the new and original richness could already be expected based on results from simple simulations. Olsen et al. (2007, Fig. 8) show that even after correcting for the redshift overestimate, the richness may be off by typically up to $50 \%$. The scatter is of the order $25 \%$. This is slightly worse than the value claimed by Postman et al. (2002).

The final corrected values for the richness are in the range 12-65 comparable to that obtained in Paper III and the range reported by Bahcall et al. (2003) using a modified version of the matched filter in their analysis of 400 square degrees of the SDSS survey. As in the previous paper we find that the measured richness and velocity dispersions follow the same relation as that determined by Bahcall et al., with comparable scatter. This again demonstrates that the simple matched-filter technique used by us does not measure richness very accurately. Furthermore, while we can use the redshift distribution to re-compute more accurate velocity dispersions in case of contamination by nearby systems, this cannot be easily done for the richness, showing that it is probably not a reliable proxy for the mass.

\subsection{Galaxy population}

Finally, we characterise the galaxy population of the confirmed clusters by studying the colour properties of the member galaxies. In particular, the colour-magnitude diagram of cluster members normally reveals the presence of a narrow sequence of bright, early-type galaxies known as the "red sequence" (e.g. Gladders et al. 1998; Stanford et al. 1998; Holden et al. 2004; López-Cruz et al. 2004), which has been used to identify clusters (e.g. Gladders \& Yee 2000). The presence of this colour-magnitude (CM) relation serves as unambiguous evidence for the presence of a real physical system. In Paper III we used this fact as the basis for an objective method for detecting red sequences for the cluster candidates identified by the use of the matched-filter method applied to single passband photometric data. While most of the previous studies focused on relatively rich systems, the presence of red sequences in very poor clusters and groups has also been reported (e.g. Andreon 2003).

For five of the ten systems analysed here, $V$-band images are available, thus allowing us to construct and investigate the CM diagram of the "cluster" members. The $(V-I) \times I$ CM diagrams were constructed considering galaxies within a radius of $0.75 h_{75}^{-1} \mathrm{Mpc}$ (the "cluster region"). The identification method is based on "tilted colour histograms", counting galaxies within slices of a given width and characterised by a slope taken to be comparable to that typically observed for the CM relations of nearby clusters (e.g. López-Cruz et al. 2004). For each cluster we construct two histograms shifted in colour by half a bin width to assure good sensitivity at any colour. For each histogram we identify the most significant peak based on the probability of finding a similar colour overdensity at a randomly chosen position. Readers are referred to Paper III for the details of the red sequence detection method.

The red sequence identification is applied separately to two samples. First, we consider the sample of all galaxies brighter than $I=20$ with and without redshifts. This has the advantage of good statistics but is susceptible to projection effects possibly leading to contamination by non-cluster members and thus dilution of the red sequence, if one exists. Second, we restrict the analysis to those galaxies for which we have redshifts. While these are not affected by projection effects, the statistics are usually poor in particular considering the sparse sampling of our survey.

The results of the analysis of the cluster CM diagrams are shown in Fig. 10. The left panels of this figure show the colourmagnitude diagrams for galaxies (dots), including all galaxies brighter than $I=21$ within the cluster region (defined above). Filled circles indicate spectroscopically confirmed members and crosses other (field) galaxies with measured redshifts. Also shown in this panel are the best-estimated loci characterising the red sequence as determined from the photometric data alone (dashed line) and that obtained considering only the confirmed spectroscopic members (solid line). The middle panels show the background-corrected colour distributions of galaxies brighter than $I=20.0$ within the cluster region. The right panels show the colour distributions of spectroscopic members (solid histograms) and that of all galaxies with measured redshifts (dotted histograms).

Using the photometric sample we find only two systems (EISJ2240-4021, \#12 and EISJ2244-4019, \#21) showing signs of a red sequence, while we identify three systems (indicated 

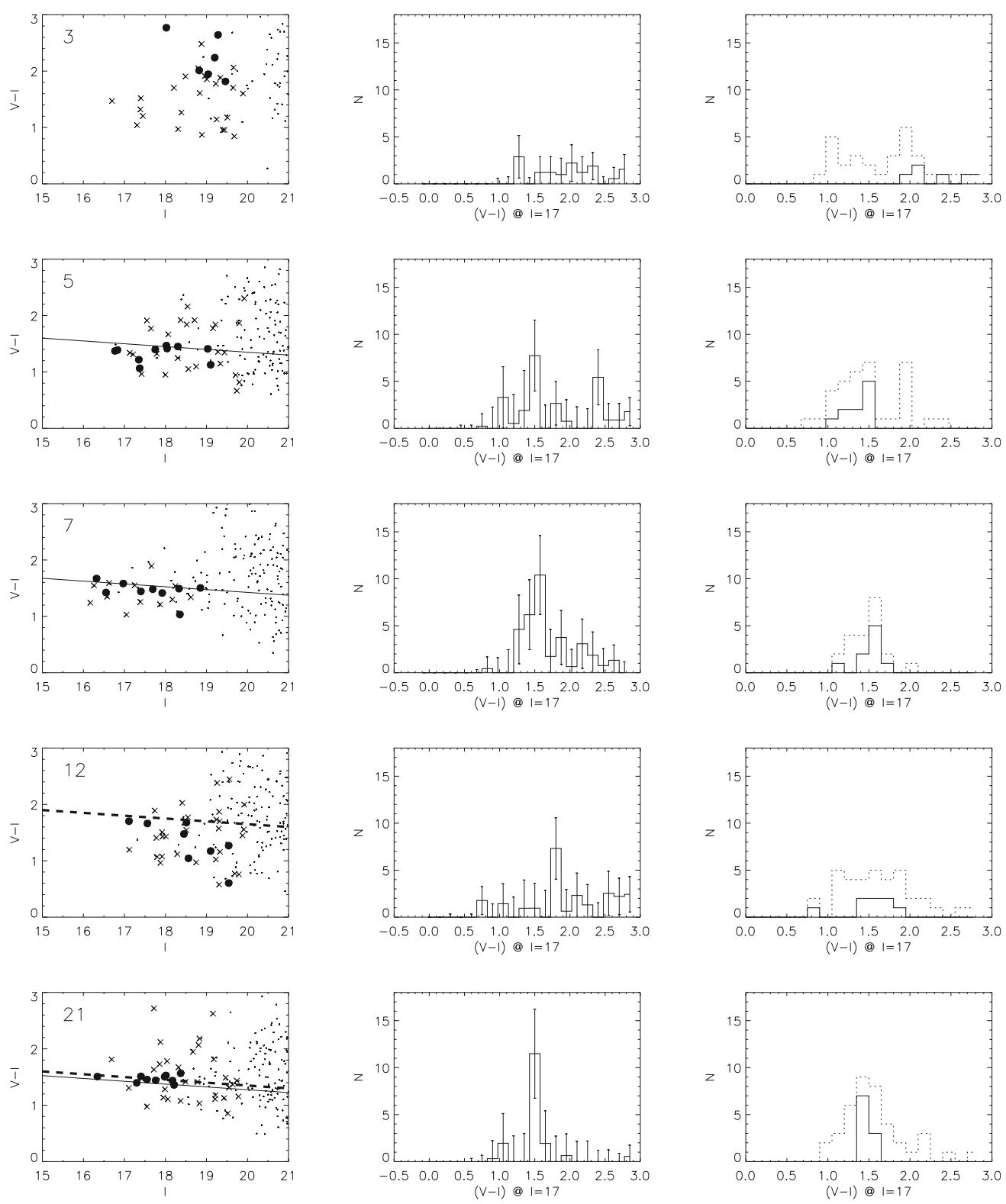

Fig. 10. For each cluster we show three diagrams with the cluster identification number indicated in the first one. The first diagram is the colourmagnitude diagram for all galaxies within $0.75 h_{75}^{-1} \mathrm{Mpc}$ from the cluster centre (dots). On top of that we mark by solid circles the spectroscopic members of the confirmed group and by crosses the remaining galaxies with redshifts. The solid line is the locus of the red sequence detected from the spectroscopic members and the dashed line is the one detected in the photometric analysis. In both cases we only show the line if we consider the sequence significant (see the text for details). The second plot is the "tilted colour histogram" for the galaxies with $I \leq 20$ in the same region statistically corrected for the background contribution. The last panel is the "tilted colour histogram" for the spectroscopic members (solid line) and for all galaxies with a redshift (dotted line).

by dashed lines in the CM diagrams of Fig. 10) when the spectroscopic sample is used. In one case (EISJ2244-4019, \#21) the red sequence is detected using both the photometric and spectroscopic samples. For EISJ2240-4021 (\#12) a red sequence is only detected from the photometric data and not from the confirmed spectroscopic members, showing the difficulties in identifying red sequences from photometric data alone.

Even though the total number of systems analysed is arguably small, the fraction of systems with a red sequence identified using the spectroscopic data is $\sim 60 \%$, similar to the fraction of 59\% reported in Paper III. However, only one system shows both a photometrically and spectroscopically identified sequence, which is significantly fewer than expected from Paper III.
In summary, a total of four systems show evidence of having a red sequence. We find two systems from the photometric data alone and three from the spectroscopic analysis with one system being in common. Both the colour and scatter of the detected red sequences are consistent with our previous work. Moreover, the colours of the galaxies along the red sequences are consistent with the passive evolution scenario for galaxy evolution. Based on our current discussion and that of Paper III we find that up to $40 \%$ of the clusters identified by the matched-filter method and confirmed spectroscopically do not have a detectable red sequence.

There are three possible explanations for the apparent lack of a red sequence in some of our systems: 1) that the systems detected by the matched-filter and tentatively confirmed 
by the spectroscopic data are just density enhancements in redshift space but do not form bound systems; 2) that spectroscopic results are biased either in the selection of targets or in the measurement of the redshift leading to a sample consisting of predominantly spiral galaxies; 3 ) that there are systems formed predominantly by a population of blue galaxies. Unfortunately, with the present data it is impossible to disentangle these various options, and must await much larger samples.

\subsection{CMDs for non-confirmed candidates}

We have also used the available colour information, to explore in more detail the fields within which we were unable to identify significant groups from the spectroscopic data possibly associated with matched-filter detections. From the 13 fields without a detection, there are 10 fields for which we have colour information. The CM diagrams for these 10 fields are shown in Fig. 11. The filled symbols represent galaxies within a radius of $0.75 h_{75}^{-1} \mathrm{Mpc}$ as defined by the redshift estimated for the matched-filter detection. The lines mark the expected position of the red sequence for redshifts $z=0.1,0.2,0.3,0.4$. The solid lines indicate the relation for the matched-filter estimated redshifts. Also shown are tilted histograms also used in the analysis of the galaxy populations of the confirmed systems. The histograms are constructed considering galaxies brighter than $I \leq 20$ and shifted by half the bin width in colour. These histograms are used to identify the most significant peaks and their $S / N$ ratios with the results summarised in Table 7 . The table gives: in Cols. 1 and 2 the field identifier and cluster name, in Cols. 3 and 5 the colour of the peaks for each of the tilted histograms; and in Cols. 4 and 6 the related $S / N$ values. From the figures and the values of $S / N$ ratio listed in Table 7 , it is clear that there are no obvious red sequences. The marginal detections of red sequences with $S / N>2$ are compared with the marginal detections of groups in redshift space listed in Table A.1. There are four such cases that are discussed below:

- for EISJ2241-4006 (\#13) the colour is found to be $V-I \sim$ 2.1 , this roughly corresponds to a redshift of $z \sim 0.4$. In the redshift distribution in Fig. 3 we find a group with six members at $z_{\text {spec }}=0.344$ with a significance of $94.5 \%$;

- for EISJ2243-4010A (\#15) the colour is found to be 2.025, which corresponds to a redshift slightly smaller than $z=0.4$. In the redshift distribution this could correspond to the group at $z=0.345$ with three members and a significance of $73.4 \%$;

- for EISJ2243-4010B (\#18) the colour is found to be 1.575 roughly corresponding to $z=0.25$. This could correspond to both groups found in the field. However, the richest group has its centre very far from the MF position and is thus unlikely to give rise to the red sequence at the MF position. The other group with three members at $z \sim 0.285$ and a significance of $80.5 \%$ is very spread over the field and from these data we cannot conclude that it is related to the MF detection;

- for EISJ2248-4015 (\#23) the colour is also 1.575 corresponding to $z \sim 0.25$. This is consistent with the group at $z=0.246$ with a significance of $98.7 \%$, which was also mentioned above as the likely origin of the MF detection.

Even though these results are by themselves inconclusive, they provide support that the matched-filter detections are, at least for the four cases listed above, indeed related to a cluster. However, a better spectroscopic coverage is needed to firmly establish the possible presence both of a cluster and a red sequence.
Table 7. The colours and $S / N$ ratio for the detected peaks in each of the tilted histograms for the non-confirmed candidates.

\begin{tabular}{rlrrrr}
\hline \hline ID & Field & $(V-I)_{1}$ & $(S / N)_{1}$ & $(V-I)_{2}$ & $(S / N)_{2}$ \\
\hline 1 & EISJ0044-2950A & 2.550 & 1.9 & 1.725 & 1.6 \\
2 & EISJ0045-2944 & 1.050 & 1.4 & 2.625 & 1.4 \\
4 & EISJ0048-2928 & 1.350 & 1.5 & 1.275 & 1.6 \\
8 & EISJ2236-4014 & 1.800 & 1.4 & 1.725 & 1.5 \\
13 & EISJ2241-4006 & 2.100 & 2.2 & 1.125 & 1.7 \\
15 & EISJ2243-4010A & 2.100 & 1.9 & 2.025 & 2.2 \\
18 & EISJ2243-4010B & 1.500 & 1.9 & 1.575 & 2.6 \\
20 & EISJ2244-4008 & 2.250 & 0.8 & 1.575 & 0.6 \\
22 & EISJ2246-4012B & 1.800 & 1.3 & 1.875 & 1.1 \\
23 & EISJ2248-4015 & 1.500 & 1.9 & 1.575 & 2.6 \\
\hline
\end{tabular}

\section{Discussion}

In this paper we present new redshifts for 747 galaxies in the fields of 23 EIS cluster candidates with redshifts $z_{\mathrm{MF}}=0.3-0.4$. In one of the fields two candidates were found, but the spectroscopic coverage was only sufficient for investigating the main target. The main aim of this spectroscopic survey was to identify overdensities in redshift space corresponding to the cluster candidates detected by the matched-filter search technique in order to evaluate the reliability of the results of this algorithm as well as investigate the characteristics of the detected systems. The combination of spectroscopic and photometric data sets available for these cluster candidates was used to characterise the confirmed systems.

For 10 of the $23(\sim 43 \%)$ targeted candidates we confirm the presence of an overdensity in redshift space. This confirmation rate is significantly smaller than that of Papers I-III, where $\sim 95 \%$ of the systems with $z_{\mathrm{MF}}=0.2$ were confirmed. This lower rate is most likely due to the brighter magnitude limit of the spectroscopic survey, which also implied probing primarily the low end of the redshift range being considered. It is worth noting, that in five of the fields observed even though no system was confirmed there was clear evidence, based on the visual inspection of the images of these fields, for the presence of a concentration of fainter galaxies at the position of the cluster candidate. Unfortunately, these fainter galaxies did not have a measured redshift. A final word on the nature of these candidates will require further observations of these fields. Furthermore, four out of five confirmed candidates with colour information show evidence for a red sequence lending further support to the interpretation that these systems are bound and have early-type galaxies in their core.

From the inspection of the colour-magnitude diagrams for 10 non-confirmed candidates suggest that four of these are possibly associated with a bound system based on the presence of a weak signal in the colour histograms matching the redshift of a marginal detection in redshift space.

Comparison of the overall properties of the sample discussed in the present paper $\left(z_{\mathrm{MF}}=0.3-0.4\right)$ and that of our previous Papers I-III $\left(z_{\mathrm{MF}}=0.2\right)$ leads to the following conclusions:

- the mean redshift of the confirmed sample is 0.24 higher than 0.18 measured in Paper III. This indicates that we have successfully identified systems at higher redshift, even though at a lower rate, which as indicated throughout the paper was mostly due to a bright limiting magnitude of the spectroscopic data; 

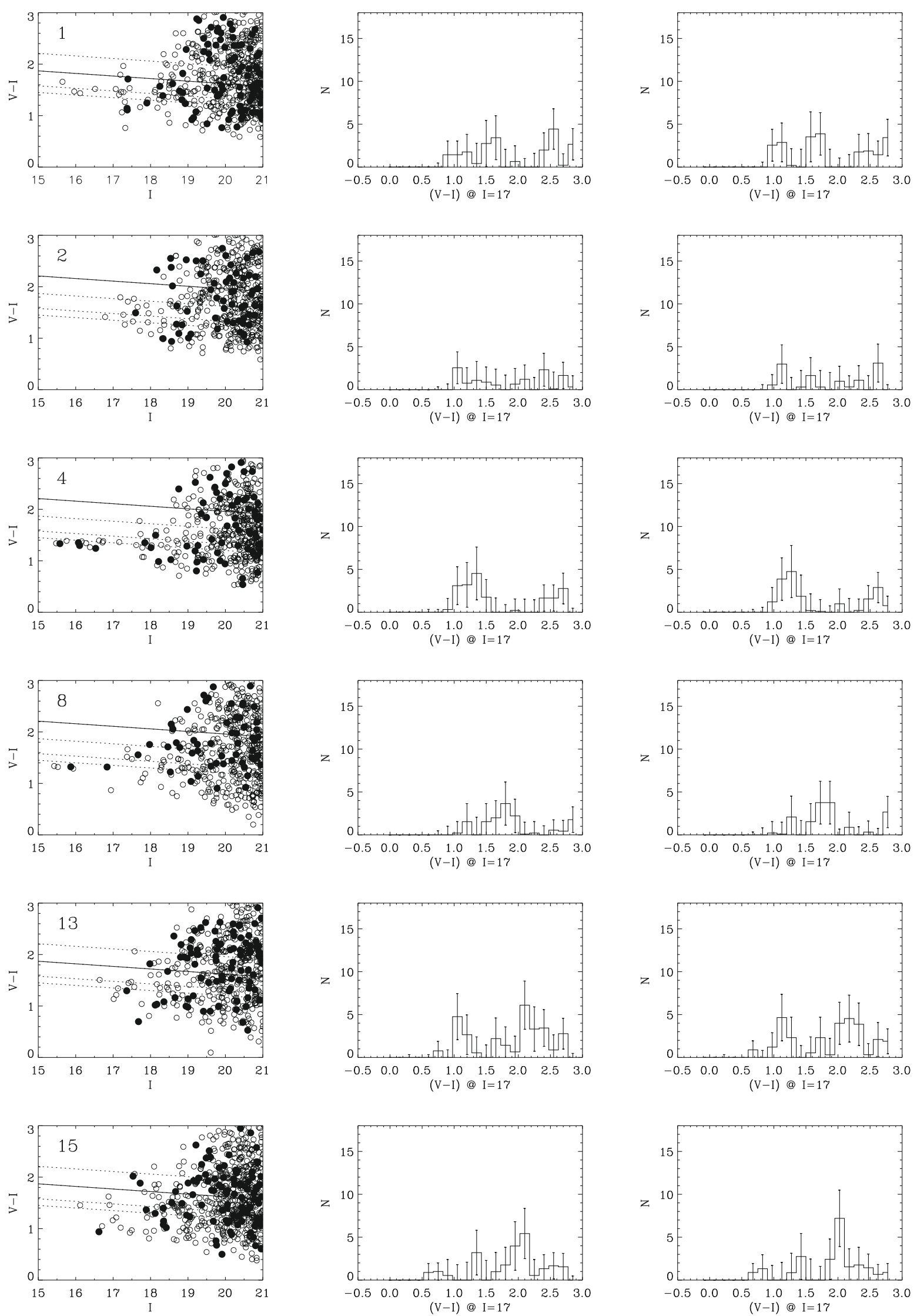

Fig. 11. Colour-magnitude diagrams for the ten non-confirmed systems with $V$ - and $I$-band data available. The filled circles mark galaxies inside the cluster region, while open circles mark objects in the background region. The lines mark the expected position of the red sequence at redshifts $0.1,0.2,0.3$ and 0.4 , with the solid line marking the one expected from the matched-filter estimated redshift. The second and third columns the two shifted, tilted histograms similar to the ones of Fig. 10 in the middle column. A magnitude cut of $I \leq 20$ is applied for constructing the tilted histograms. 

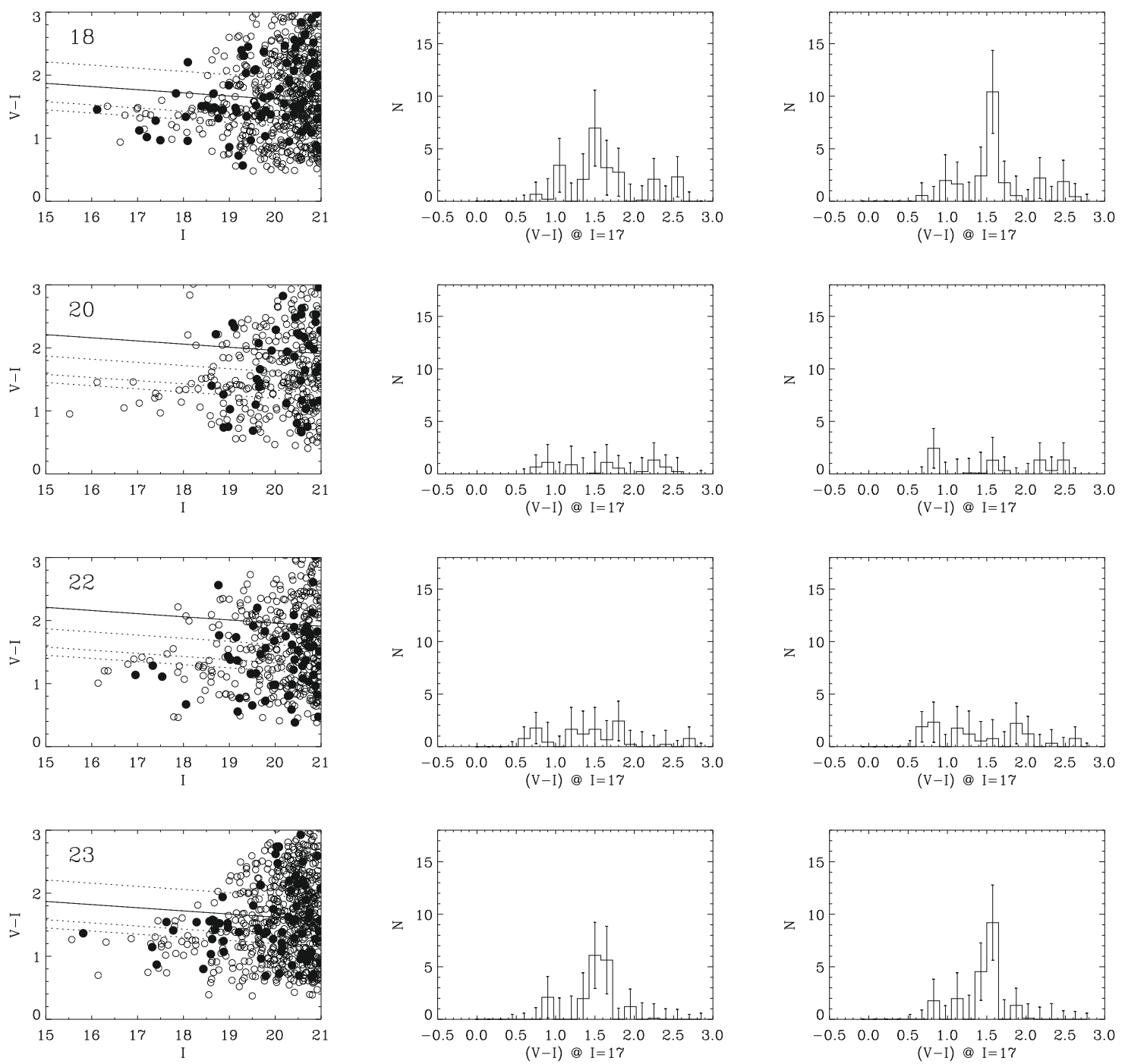

Fig. 11. continued.

- consistent with our findings in Paper III, $\sim 60 \%$ of the identified overdensities in redshift space with photometric data in two passbands show evidence for a red sequence among their spectroscopic members with a colour consistent with their measured redshift. This lends credence to the interpretation that these overdensities are indeed bound systems with a well-defined population of early-type galaxies. On the other hand, the remaining cases may not be totally discarded as they may still be spiral-rich bound systems. A final conclusion will require a considerable improvement in sampling, a hard task considering the angular extent of these systems;

- the distribution of velocity dispersions is consistent with that obtained by the much larger SDSS survey;

- the range of richness of our confirmed systems is in good agreement with those of Bahcall et al. (2003) analysing a subset of the SDSS data using a similar implementation of the matched-filter algorithm. As previous authors we find that richness as estimated by the matched filter is not an adequate proxy for the mass of the system.

\section{Summary}

In this series of four papers we have measured 1954 galaxy redshifts in the range $0.0065-0.6706$ over 58 fields around EIS candidate clusters identified using the matched-filter algorithm and with estimated redshifts below $z_{\mathrm{MF}}=0.4$. For a total of 42 cases we were able to associate the candidate with density enhancements in redshift space with mean redshifts between 0.095 and 0.534 . This represents a yield of $\sim 75 \%$ for the matched-filter technique, which is consistent with the original estimates by Olsen et al. (1999a) based on simulations and also comparable to the estimates reported by Kim et al. (2002). The method tends to overestimate the system redshift reaching an offset of 0.1 at $z_{\mathrm{MF}} \lesssim 0.4$. The number of galaxies with concordant redshifts range from four to 35 . The one-dimensional velocity dispersions of the identified density enhancements, which we have referred to as systems, vary from very low values up to $\sim 1400 \mathrm{~km} \mathrm{~s}^{-1}$, with the equivalent number of $L^{*}$ galaxies varying from 11 to 65. Due to the undersampling of the systems by our spectroscopic observations, these values, in general, have large errors and cannot by themselves distinguish between chance enhancements, small groups and rich clusters. Based on the results of this series we find that the identified clusters consist of substructures in more than $35 \%$ of the cases, impacting the matched-filter richness estimates.

For 34 of the confirmed groups and clusters colour information is available and was used to search for a red sequence. For 20 systems, corresponding to $\sim 60 \%$, we were able to detect one 
using the spectroscopically confirmed members. While having a red sequence is not a necessary condition for a bound system, its existence definitely lends further credence to the detection. On the other hand, the matched-filter technique may detect systems with a large fraction of late-type galaxies or where the early-type galaxies are affected by recent starbursts that may occur during merging of groups or clusters. The confirmation of this idea will require more extensive spectroscopic surveys than the one carried out in the present program.

This series complements the work carried out by Ramella et al. (2000), Benoist et al. (2002), Olsen et al. (2005b) who carried out spectroscopic observations of EIS candidate clusters at intermediate and high redshifts, obtaining confirmation yields, redshift offsets relative to the matched-filter estimates and velocity dispersions similar to those reported here.

Combining the results of this series with those of the previous papers, we have obtained roughly 2500 redshifts for a total of 74 cluster candidate fields out of 302 in the original candidate list, spanning the redshift range from 0.2 to 1.3 , being perhaps one of the most extensive spectroscopic follow-up works of its kind. Taken altogether, these results show that the matched-filter technique leads to the detection of real density enhancements in redshift space. It should be used in conjunction with other cluster search techniques for the optimal identification of clusters of galaxies from optical and near-infrared imaging data. This is extremely important considering that large surveys such as UKIDSS, Dark Energy Survey and VISTA are either on-going or are envisioned to start in the next few years providing unprecedented samples of candidate clusters. Understanding the differences and possible biases of different detection techniques is particularly important for determining the completeness of the observed samples and be able to use, for instance, number counts of clusters of galaxies as a tool to constrain cosmological parameters as proposed by several new dark energy projects.

On-going and future surveys will provide considerable more information enabling the application of other detection algorithms based on for instance colours or photometric redshifts. However, regardless of the detection algorithm optical cluster surveys will always have to rely on extensive spectroscopic follow-up both for confirmation, membership assignment, subclustering and for fully characterising the systems. Some of these issues are addressed in the present work.

Acknowledgements. We thank the anonymous referee for useful comments which greatly helped improving the paper. We thank John Pritchard, Lisa Germany and Ivo Saviane for making the pre-imaging observations of the fields. We would also like to thank the $2 \mathrm{p} 2$ team, La Silla, for their support at any time during the observations. We are also in debt to Morten Liborius Jensen for preparing the slit masks. This research has made use of the NASA/IPAC Extragalactic Database (NED) which is operated by the Jet Propulsion Laboratory, California Institute of Technology, under contract with the National Aeronautics and Space Administration. This work has been supported by The Danish Board for Astronomical Research. LFG acknowledges financial support from the Carlsberg Foundation and the Danish Natural Science Research Council. The Dark Cosmology Centre is funded by the Danish National Research Foundation.

\section{Appendix A: Marginal detections of groups in redshift}

This appendix presents the 46 groups identified in redshift space but which do not fulfil our significance criterion. These groups are listed here to help the discussion of Sect. 4.3. Table A.1 gives in Cols. 1 and 2 the field identifier and the cluster field name; in Col. 3 the number of spectroscopic members of the group; in Cols. 4 and 5 the mean position in J2000; in Col. 6 the mean redshift of the group members; in Col. 7 the velocity dispersion corrected for our measurement accuracy. In cases where the measured velocity dispersion is smaller than the measurement error we list the value of $\sigma_{v}=0$; in Col. 8 the significance as defined above and in Col. 9 the distance in arcmin between the group and the original MF position.

\section{Appendix B: Non-confirmed candidates with significant redshift detections}

This appendix presents detailed descriptions for the nine fields within which a significant group was identified but not associated to the EIS detection.

- EISJ0045-2944 (\#2): the identified group in redshift space covers a large area of the field and shows no concentration at the expected position. Therefore, it was discarded as a confirmation. From the image it is likely that the four galaxies of similar brightness located at the centre of the field are responsible for the signal. Note that the two redshifts measured in this group do not agree.

- EISJ0048-2928 (\#4): in this field two groups were found, both at quite low redshift with the nearest group being located almost at the position of the matched-filter detection. However, inspecting the image one finds in the same region a handful of galaxies with magnitudes $I \sim 19$ matching the Schechter magnitude at $z=0.4$. We believe that the latter group is the one responsible for the detection. A firm conclusion about this candidate will require considerable more data.

- EISJ2236-4014 (\#8): there is only one significant group, but it is poor, spread over the field and far away from the centre. From the image it can be seen that there are no redshifts obtained for the galaxies close to the position of the matchedfilter detection. There are a number of galaxies with magnitudes matching those expected for a group at the cluster candidate estimated redshift.

- EISJ2241-3932 (\#14): in this field a significant group with three members is found to the east of the matched-filter position. From the image a promising concentration of galaxies is found at the matched-filter position. We conclude that there could be a cluster but more spectroscopic data are needed for a firm conclusion.

- EISJ2243-3947 (\#19): three significant groups are identified, all at quite low redshift. The richest group with seven members is located to the east of the cluster position. The two other groups have three and four members with the smallest one also being displaced to the west of the centre of the matched-filter detection. Inspecting the image we find a compact group of galaxies most likely causing the matched filter detection. Only one of these galaxies have a redshift measured at $z=0.2301$, thus not belonging to any of the identified groups in redshift space. It is thus inconclusive whether this is the true redshift of the concentration or whether it is caused by chance alignment.

- EISJ2243-3952 (\#16): two significant groups are found, both of them spread over the entire field with no clear concentration. From the image it can be seen that this detection is close to the position of a nearby galaxy. We believe that spurious objects found in the vicinity of this bright galaxy has caused the MF detection. We consider this detection totally spurious and an artifact caused by problems in the original galaxy catalogue. 
Table A.1. Identified groups with a significance less than $99 \%$.

\begin{tabular}{|c|c|c|c|c|c|c|c|c|}
\hline ID & Cluster field ${ }^{a}$ & Members & $\alpha(\mathrm{J} 2000)$ & $\delta(\mathrm{J} 2000)$ & $z$ & $\sigma_{v}\left[\mathrm{~km} \mathrm{~s}^{-1}\right]^{b}$ & $\sigma_{1}[\%]$ & Dist. ['] \\
\hline 1 & EISJ0044-2950A & 3 & 004449.9 & -295004.0 & 0.173 & 0 & 98.0 & 2.0 \\
\hline 1 & EISJ0044-2950A & 3 & 004445.5 & -295038.8 & 0.259 & 444 & 91.3 & 2.9 \\
\hline 2 & EISJ0045-2944 & 4 & 004507.1 & -294536.3 & 0.186 & 627 & 97.9 & 1.5 \\
\hline 2 & EISJ0045-2944 & 3 & 004509.0 & -294449.6 & 0.226 & 0 & 84.9 & 1.8 \\
\hline 2 & EISJ0045-2944 & 4 & 004507.6 & -294531.9 & 0.373 & 398 & 80.2 & 1.6 \\
\hline 3 & EISJ0047-2942 & 3 & 004714.1 & -294313.3 & 0.217 & 0 & 82.3 & 1.9 \\
\hline 3 & EISJ0047-2942 & 5 & 004729.4 & -294306.1 & 0.264 & 747 & 98.9 & 1.4 \\
\hline 3 & EISJ0047-2942 & 4 & 004735.4 & -294446.5 & 0.335 & 386 & 90.7 & 3.2 \\
\hline 5 & EISJ0049-2920 & 3 & 004934.9 & -292015.7 & 0.293 & 187 & 81.8 & 0.8 \\
\hline 5 & EISJ0049-2920 & 5 & 004924.3 & -292027.5 & 0.325 & 163 & 97.0 & 1.5 \\
\hline 7 & EISJ2236-4026 & 3 & 223638.7 & -402801.8 & 0.153 & 37 & 98.3 & 2.4 \\
\hline 7 & EISJ2236-4026 & 3 & 223626.1 & -402729.6 & 0.194 & 263 & 92.4 & 4.2 \\
\hline 8 & EISJ2236-4014 & 5 & 223649.1 & -401522.8 & 0.274 & 579 & 98.7 & 0.8 \\
\hline 8 & EISJ2236-4014 & 3 & 223641.6 & -401547.3 & 0.373 & 510 & 67.0 & 2.3 \\
\hline 9 & EISJ2237-4000 & 4 & 223719.7 & -395806.6 & 0.242 & 147 & 93.8 & 2.7 \\
\hline 9 & EISJ2237-4000 & 5 & 223722.1 & -400050.6 & 0.270 & 662 & 98.8 & 2.1 \\
\hline 10 & EISJ2238-3934 & 3 & 223817.3 & -393534.4 & 0.145 & 564 & 96.6 & 2.8 \\
\hline 11 & EISJ2239-3954 & 4 & 223926.3 & -395327.8 & 0.233 & 468 & 92.8 & 1.9 \\
\hline 12 & EISJ2240-4021 & 3 & 224011.7 & -402509.6 & 0.115 & 135 & 97.7 & 4.1 \\
\hline 12 & EISJ2240-4021 & 3 & 224007.7 & -402259.2 & 0.291 & 593 & 79.0 & 1.9 \\
\hline 13 & EISJ2241-4006 & 3 & 224115.4 & -400749.3 & 0.196 & 503 & 90.0 & 2.6 \\
\hline 13 & EISJ2241-4006 & 5 & 224131.6 & -400758.2 & 0.246 & 165 & 96.5 & 1.8 \\
\hline 13 & EISJ2241-4006 & 3 & 224128.7 & -400630.2 & 0.294 & 939 & 83.2 & 0.4 \\
\hline 13 & EISJ2241-4006 & 6 & 224140.4 & -400827.6 & 0.344 & 147 & 94.4 & 3.3 \\
\hline 13 & EISJ2241-4006 & 3 & 224113.5 & -400626.7 & 0.531 & 146 & 98.5 & 2.5 \\
\hline 15 & EISJ2243-4010A & 3 & 224248.4 & -401052.0 & 0.150 & 142 & 98.4 & 2.6 \\
\hline 15 & EISJ2243-4010A & 3 & 224314.6 & -400903.0 & 0.216 & 398 & 83.9 & 2.8 \\
\hline 15 & EISJ2243-4010A & 3 & 224304.7 & -401005.4 & 0.345 & 313 & 73.4 & 0.6 \\
\hline 16 & EISJ2243-3952 & 3 & 224312.4 & -395348.7 & 0.171 & 70 & 97.5 & 1.7 \\
\hline 16 & EISJ2243-3952 & 5 & 224308.2 & -395313.2 & 0.245 & 70 & 95.0 & 2.2 \\
\hline 17 & EISJ2243-3959 & 5 & 224340.2 & -400030.9 & 0.214 & 357 & 94.8 & 2.3 \\
\hline 17 & EISJ2243-3959 & 4 & 224345.4 & -395958.3 & 0.259 & 796 & 94.1 & 3.1 \\
\hline 17 & EISJ2243-3959 & 3 & 224330.5 & -400103.8 & 0.347 & 568 & 81.6 & 1.5 \\
\hline 18 & EISJ2243-4010B & 3 & 224344.4 & -401200.5 & 0.285 & 0 & 80.5 & 1.5 \\
\hline 19 & EISJ2243-3947 & 5 & 224355.6 & -394742.7 & 0.196 & 545 & 98.5 & 0.2 \\
\hline 19 & EISJ2243-3947 & 3 & 224334.5 & -394826.1 & 0.341 & 421 & 79.3 & 4.2 \\
\hline 20 & EISJ2244-4008 & 3 & 224429.8 & -400844.2 & 0.178 & 501 & 96.2 & 1.6 \\
\hline 20 & EISJ2244-4008 & 3 & 224452.4 & -400851.9 & 0.199 & 0 & 88.5 & 5.9 \\
\hline 20 & EISJ2244-4008 & 5 & 224410.8 & -400756.5 & 0.213 & 383 & 96.9 & 2.1 \\
\hline 20 & EISJ2244-4008 & 3 & 224411.2 & -400719.6 & 0.359 & 681 & 78.5 & 2.3 \\
\hline 21 & EISJ2244-4019 & 3 & 224432.6 & -402100.4 & 0.228 & 182 & 83.9 & 1.5 \\
\hline 21 & EISJ2244-4019 & 4 & 224457.2 & -401756.2 & 0.244 & 632 & 92.3 & 5.8 \\
\hline 21 & EISJ2244-4019 & 6 & 224418.4 & -402040.1 & 0.340 & 431 & 93.9 & 2.1 \\
\hline 22 & EISJ2246-4012B & 3 & 224640.6 & -401311.6 & 0.126 & 138 & 98.4 & 1.6 \\
\hline 22 & EISJ2246-4012B & 3 & 224705.2 & -401429.3 & 0.148 & 405 & 98.6 & 3.6 \\
\hline 23 & EISJ2248-4015 & 6 & 224857.9 & -401540.5 & 0.246 & 567 & 98.7 & 0.7 \\
\hline
\end{tabular}

${ }^{a}$ Fields where no additional marginal detections were found are not included in the table; ${ }^{b} \sigma_{v}=0$ reflects a measured velocity dispersion below the measurement error.

- EISJ2243-4010B (\#18): only one significant group is found with a centre very far from the original matched filter position. From the image there is a sparse group of galaxies at the position of the matched filter detection, however only a foreground galaxy has a redshift measured.

- EISJ2244-4008 (\#20): we find two significant groups. Both of these are wide spread and displaced from the original matched filter position. The concentration of galaxies at the matched filter position all match the expected magnitudes but the obtained redshifts vary a lot. We therefore conclude that there is likely no cluster at this position.

- EISJ2248-4015 (\#23): in this field three groups were detected - two are significant and listed in Table 4 and one less significant listed in Table A.1. All of them have redshifts ranging from 0.13 to 0.25 making the association with the matched filter detection difficult. We do not believe that the significant groups are associated with the matched filter detection as they cover the entire field of view. On the other 
hand, from the image we find that the less significant group (at 98.6\%) forms a compact system very close to the matched filter position, with six galaxies having concordant redshifts near $z=0.246$. We, therefore, believe that this system is responsible for the detection. This conclusion is supported by the analysis of the colour-magnitude diagram in Sect. 4.3.

\section{References}

Andreon, S. 2003, A\&A, 409, 37

Andreon, S. 2008, MNRAS, 386, 1045

Bahcall, N., McKay, T., Annis, J., et al. 2003, ApJS, 148, 243

Becker, M. R., McKay, T. A., Koester, B., et al. 2007, ApJ, 669, 905

Beers, T., Flynn, K., \& Gebhardt, K. 1990, AJ, 100, 32

Benoist, C., da Costa, L., Olsen, L. F., et al. 1999, A\&A, 346, 58

Benoist, C., da Costa, L., Jørgensen, H., et al. 2002, A\&A, 394, 1

Böhringer, H., Voges, W., Huchra, J. P., et al. 2000, ApJS, 129, 435

Buote, D. A., \& Tsai, J. C. 1996, ApJ, 458, 27

Gal, R. R., Lemaux, B. C., Lubin, L. M., Kocevksi, D., \& Squires, G. K. 2008, ArXiv e-prints, 803

Gilbank, D. G., Bower, R. G., Castander, F. J., \& Ziegler, B. L. 2004, MNRAS, 348,551

Gladders, M., \& Yee, H. 2000, AJ, 120, 2148

Gladders, M., \& Yee, H. 2001, in The New Era of Wide Field Astronomy (ASP), ASP Conf. Ser., 232, 126

Gladders, M., López-Cruz, O., Yee, H., \& Kodama, T. 1998, ApJ, 501, 571

Gonzalez, A., Zaritsky, D., Dalcanton, J., \& Nelson, A. 2001, ApJS, 137, 117
Gunn, J., Hoessel, J., \& Oke, J. 1986, ApJ, 306, 30

Hansen, L., Olsen, L. F., \& Jørgensen, H. 2002, A\&A, 388, 1 (Paper I)

Holden, B., Nichol, R., Romer, A., et al. 1999, AJ, 118, 2002

Holden, B., Stanford, S., Eisenhardt, P., \& Dickinson, M. 2004, AJ, 127, 2484

Jeltema, T. E., Hallman, E. J., Burns, J. O., \& Motl, P. M. 2008, ApJ, 681, 167

Katgert, P., Mazure, A., Perea, J., et al. 1996, A\&A, 310, 8

Kim, R., Kepner, J., Postman, M., et al. 2002, AJ, 123, 20

Kinney, A., Calzetti, D., Bohlin, R., et al. 1996, ApJ, 467, 38

Koester, B. P., McKay, T. A., Annis, J., et al. 2007, ApJ, 660, 239

López-Cruz, O., Barkhouse, W. A., \& Yee, H. K. C. 2004, ApJ, 614, 679

Nonino, M., Bertin, E., da Costa, L., et al. 1999, A\&AS, 137, 51

Olsen, L. F. 2000, Ph.D. Thesis, Copenhagen University Observatory

Olsen, L. F., Scodeggio, M., da Costa, L., et al. 1999a, A\&A, 345, 681

Olsen, L. F., Scodeggio, M., da Costa, L., et al. 1999b, A\&A, 345, 363

Olsen, L. F., Hansen, L., Jørgensen, H., et al. 2003, A\&A, 409, 439 (Paper II)

Olsen, L. F., Benoist, C., da Costa, L., Hansen, L., \& Jørgensen, H. 2005a, A\&A,

435, 781, Paper III

Olsen, L. F., Zucca, E., Bardelli, S., et al. 2005b, A\&A, 442, 841

Olsen, L. F., Benoist, C., Cappi, A., et al. 2007, A\&A, 461, 81

Postman, M., Lubin, L., Gunn, J., et al. 1996, AJ, 111, 615

Postman, M., Lauer, T., Oegerle, W., \& Donahue, M. 2002, ApJ, 579, 93

Prandoni, I., Wichmann, R., da Costa, L., et al. 1999, A\&A, 345, 448

Ramella, M., Biviano, A., Boschin, W., et al. 2000, A\&A, 360, 861

Rosati, P. 1998, Astron. Nachr., 319, 79

Scodeggio, M., Olsen, L. F., da Costa, L., et al. 1999, A\&AS, 137, 83

Stanford, S., Eisenhardt, P., \& Dickinson, M. 1998, ApJ, 492, 461

van Breukelen, C., Clewley, L., Bonfield, D. G., et al. 2006, MNRAS, 373, L26

Yee, H., Morris, S., Lin, H., et al. 2000, ApJS, 129, 475

Zandivarez, A., Martínez, H., \& Merchán, M. 2006, ApJ, 650, 137 